1 Functional characterization of 5' UTR cis-acting sequence elements that modulate translational

2 efficiency in $P$. falciparum and humans

3

4 Valentina E. Garcia ${ }^{1}$, Rebekah Dial ${ }^{1,2}$, Joseph L. DeRisi*1,3

$5{ }^{1}$ Univeristy of California, San Francisco, ${ }^{2}$ Denali Therapeutics, ${ }^{3}$ Chan Zuckerberg Biohub

$6 \quad *$ Corresponding Author

$7 \quad$ Abstract

8 Background

9 The eukaryotic parasite Plasmodium falciparum causes millions of malarial infections

10 annually while drug resistance to common antimalarials is further confounding eradication

11 efforts. Translation is an attractive therapeutic target that will benefit from a deeper

12 mechanistic understanding. As the rate limiting step of translation, initiation is a primary driver

13 of translational efficiency. It is a complex process regulated by both cis and trans acting factors,

14 providing numerous potential targets. Relative to model organisms and humans, $P$. falciparum

15 mRNAs feature unusual 5' untranslated regions suggesting cis-acting sequence complexity in

16 this parasite may act to tune levels of protein synthesis through their effects on translational

17 efficiency.

18 Methods

19 Here, we deployed in vitro translation to compare the role of cis-acting regulatory

20 sequences in P. falciparum and humans. Using parasite mRNAs with high or low translational

21 efficiency, the presence, position, and termination status of upstream "AUG" $s$, in addition to

22 the base composition of the $5^{\prime}$ untranslated regions, were characterized.

\title{
23 Results
}


The density of upstream "AUG" $s$ differed significantly among the most and least

25 efficiently translated genes in P. falciparum, as did the average " $G C$ " content of the 5'

26 untranslated regions. Using exemplars from highly translated and poorly translated mRNAs,

27 multiple putative upstream elements were interrogated for impact on translational efficiency.

28 Upstream "AUG"s were found to repress translation to varying degrees, depending on their

29 position and context, while combinations of upstream "AUG"s had nonadditive effects. The

30 base composition of the $5^{\prime}$ untranslated regions also impacted translation, but to a lesser

31 degree. Surprisingly, the effects of cis-acting sequences were remarkably conserved between $P$.

32 falciparum and humans.

\section{Conclusion}

While translational regulation is inherently complex, this work contributes toward a

more comprehensive understanding of parasite and human translational regulation by

36 examining the impact of discrete cis-acting features, acting alone or in context.

\section{Keywords}

38 In vitro translation, translation initiation, upstream "AUG"s, upstream open reading frames

\section{Background}

As the primary cause of severe malaria, Plasmodium falciparum remains a major global

41 health threat. In 2018, approximately 228 million cases of malaria led to 405,000 deaths,

42 primarily of children under the age of 5 [1]. Control and eradication of $P$. falciparum is

43 complicated by widespread or emerging drug resistance to all common antimalarial drugs [2-

44 4]. To circumvent drug resistance, targeted therapeutic development has the potential to

45 generate novel antimalarials with unique mechanisms of action. Unfortunately, targeted 
46 development is hindered by an incomplete understanding of the basic molecular processes of

$47 \quad$ P. falciparum and how they differ from human biology.

Recently, translation has emerged as a potentially druggable pathway [5-7]. While no clinically approved antimalarials target cytoplasmic translation [5], there are promising new

51 candidates to distinct translational mechanisms. For example, there is a growing number of

52 compounds targeting tRNA synthetases [8,9], M5717 (formerly DDD107498) is currently in

53 human trials and inhibits eukaryotic elongation factor $2[6,8]$, and MMV008270 has been shown

54 to selectively inhibit parasite translation through an unknown mechanism of action [10].

55 Currently no candidates are known to target translation initiation.

Eukaryotic translation initiation determines the rate of translation of a given mRNA,

58 referred to as the translational efficiency $(\mathrm{TE})[11,12]$. Initiation at the proper translation start

59 site (typically an "AUG" start codon) relies on interactions between the start codon and the

60 local sequence context (the Kozak sequence) with the initiator Met-tRNA and other initiation

61 factors [13-15]. TE can additionally be regulated by cis-acting sequence elements throughout

62 the $5^{\prime}$ untranslated region ( $5^{\prime}$ UTR), the sequence proceeding the translation start site. In

63 particular, upstream "AUG"s (UAUGs) are commonly observed regulatory features that are

64 divided into two groups: those that initiate open reading frames that extend beyond the

65 translation initiation site, and those that are terminated, meaning they form upstream open

66 reading frames (UORFs) by having an in-frame stop site proceeding the protein coding region

67 [16-18]. These cis-acting regulatory elements lower TE through many potential mechanisms 
68 including by initiating translation out of frame from the downstream ORF, by adding long amino

69 acid extensions at the N-terminus, or by sequestering ribosomes within the 5' UTRs [19-21].

A well-documented example of UAUG/uORF driven regulation is GCN4 in Saccharomyces

71 cerevisiae. The 5' UTR of GCN4 contains four short uORFs that themselves are differentially

72 translated under conditions of stress. Based on the availability of translation initiation factors,

73 the uORFs modulate the translation rate of the primary protein coding region to fit the

74 organisms current nutrient conditions $[22,23]$. While this example is deeply understood, it is

75 not broadly generalizable, and the rules by which such sequences exert influence on TE remain

76 challenging to describe even for the most studied of eukaryotes. For example, numerous

77 variables have been identified in other contexts that modulate the effect of uAUGs and uORFs,

78 including the Kozak sequence of the UAUG itself and the reading frame relative to the

79 translational start site $[19,24]$.

80 Studies of $P$. falciparum have confirmed that it possesses the expected eukaryotic cap-

81 binding factors required for cap-dependent translation initiation [25,26]. Additionally, gene

82 specific studies show that UAUGs and UORFs can repress translation in $P$. falciparum and that

83 the Kozak sequence of UAUGs along with uORF length may modulate their effect on TE $[27,28]$.

84 This is particularly intriguing since $P$. falciparum has repeatedly been shown to have unusually

85 long 5' UTRs containing many uAUGs $[18,29,30]$. Together this suggests that multiple cis-acting

86 factors within the 5' UTRs of $P$. falciparum could act broadly to tune TE throughout the normal

87 lifecycle, as opposed to regulating specific genes under extreme conditions, such as with GCN4

88 regulation. However, extensive ribosome profiling from our lab revealed that transcription and

89 translation rates are highly correlated throughout the intraerythrocytic life cycle with less than 
$10 \%$ of the transcriptome being under significant translational control [18]. Ribosome profiling

91 also showed that the presence of UAUGs and UORFs did not appear to correlate with TE, which

92 is in contrast to model organisms and classic paradigms like yeast GCN4. Together this

93 highlights that it remains difficult to predict how cis-acting sequences within a given $5^{\prime}$ UTR will

94 affect TE, especially in disparate eukaryotic species.

97 regulatory elements with respect to TE in both $P$. falciparum and human cells through a highly

99 developed an equivalent assay for human K562 cells. Using a pair of naturally occurring $P$.

100 falciparum 5' UTRs with differing TEs, the individual contributions of the sequence context,

101 positionality, and termination status of UAUGs, along with the base composition of the 5' UTR

102 to TE, were systematically dissected to understand their contributions, in isolation and in

103 combination. Together these data present a complex portrait of interacting elements within $5^{\prime}$

104 UTRs that directly influence TE, most of which are similar in both $P$. falciparum and human.

\section{Methods}

107

Identifying characteristics associated with high and low TE from the 5' UTRs of $P$. falciparum

The ribosome profiling and $\mathrm{mRNA}$ sequencing data from the late trophozoite stage

111 generated by Caro and Ahyong et. al. 2014 [18] were filtered for an abundance above 32 reads

112 per million, a TE greater than zero, and a predicted 5' UTR length above 175 nucleotides.

113 Additionally, 30 genes that are not included in the PlasmoDB-28 P. falciparum 3D7 gene 
114 annotations were removed. This resulted in a data set containing 2088 genes (Additional File 1).

115 The 5' UTR sequences were determined using the PlasmoDB-28 P. falciparum 3D7 genome.

116 Sequence analysis was done using Python, K.S. tests were done using the Python SciPy package,

117 and the data for Figure 1 was graphed using the Python Matplotlib package.

Cloning length variations of PF3D7_1411400 and PF3D7_1428300 5' UTRs

121 construct without a 5' UTR (Additional File 2). Using In-fusion cloning the 5' UTR and firefly

122 luciferase enzyme from the EBA175-Firefly plasmid used previously $[5,10]$ were replaced with

123 the NanoLuc Luciferase (Promega) coding sequence. The plasmid generated, called P16,

124 consists of: Puc118 backbone with a T7 promotor proceeding the NanoLuc Luciferase protein 125 coding sequence followed by the 3' UTR from PF_HRP2.

128 and PF3D7_1428300 were amplified from P. falciparum W2 strain gDNA using Kapa 2G Robust

129 DNA polymerase (Roche KK5024) with primers containing overhangs with the T7 promoter

130 (forward primer) or NanoLuc (reverse primer). The P16 plasmid was amplified using Phusion

131 polymerase (NEB M0530S) for the backbone (forward primer: ATGGTCTTCACACTCGAAGATTTC,

132 reverse primer: CCTATAGTGAGTCGTATTAGAATTCG). The inserts and backbone were purified

133 using a Zymo DNA Clean and Concentrator-5 (Zymo Research D4013). In-fusion reactions were

134 performed per the In-fusion Cloning Kit (Takara 638918) instructions and reactions were

135 transformed into Stellar Competent Cells (Takara 636766). 
137 Cloning 5' UTR 130 nucleotide constructs

To generate the 130 nucleotide 5' UTR constructs, long oligos containing an EcoRI-HF

139 cut site, the T7 promoter, the desired 5' UTR sequence, and a priming sequence to NanoLuc

140 were purchased from Integrated DNA Technologies (forward primer:

141 TGATTACGAATTCTAATACGACTCACTATAGG- desired 5' UTR - ATGGTCTTCACACTCGAAGATTTC).

142 The P16 plasmid was used as a template for PCR using Kapa 2G Robust with the reverse primer

143 binding just after the BamHI-HF restriction site in Puc118 (reverse primer:

144 CTGCAGGTCGACTCTAGA). PCR products were run on a $1 \%$ agarose gel to check the product size

145 and purified using Zymo DNA Clean and Concentrator-5 (Zymo Research D4013). To create the

146 cloning insert, purified PCR product was digested with EcoRI-HF and BamHI-HF at $37^{\circ} \mathrm{C}$ for 1.5

147 hours and purified again using a Zymo DNA Clean and Concentrator-5 (Zymo Research D4013).

148 For the cloning backbone, P16 was digested with EcoRI-HF and BamHI-HF at room temperature

149 overnight ( 12 hours), run on a 1\% agarose gel, and gel extracted with the Zymoclean Gel DNA

150 Recovery Kit (Zymo Research D4008). The insert and backbone were ligated using T4 DNA ligase

151 (NEB M0202S) at room temperature for 30 mins and heat inactivated at $65^{\circ} \mathrm{C}$ for 10 mins. After

152 heat inactivation, the reaction was transformed into Stellar Competent Cells (Takara 636766).

153 All constructs were sequence verified. The sequences for all the 5' UTRs evaluated can be found

154 in Additional File 3.

155

156 Generating reporter RNA for in vitro translation 
All mRNA generating plasmids were digested with Pvull-HF (NEB R[ $\Delta 4] 151 \mathrm{~L})$ and ApaLI-

$\mathrm{HF}$ (NEB R0507L) at $37^{\circ} \mathrm{C}$ for 3 hours. After digestion, templates were run on a $1 \%$ agarose gel to confirm cutting and the reactions were purified with Zymo DNA Clean and Concentrator-5

160 (Zymo Research D4013). 1ug of linearized template was used in a 100uL T7 RNA Polymerase

161 (purified in house) reaction that was incubated at $37^{\circ} \mathrm{C}$ for three hours. After T7 reactions were

162 complete, 15uL TurboDNAse (ThermoFisher Scientific AM2238) was added, and reactions were

163 incubated at $37^{\circ} \mathrm{C}$ for 15 minutes. The RNA was then purified using a Zymo RNA Clean and

164 Concentrator-25 Kit (Zymo Research R1017). Eluted RNA was measured using the Qubit RNA HS 165 Assay Kit (Thermo Fisher Scientific Q32852) then capped following the protocol for the Vaccinia

166 Capping System (NEB M2080S) and purified one last time using Zymo RNA Clean and

167 Concentrator-5 (Zymo Research R1013). Capped RNA concentrations were measured using the

168 Qubit RNA HS Assay Kit (Thermo Fisher Scientific Q32852). Final RNA was diluted to

$1690.25 \mathrm{pmoles} / \mathrm{ul}$ for use in the in vitro translation assays.

170 For the comparing capped veruses uncapped mRNA, uncapped RNA was incubated for 5

171 minutes at $65^{\circ} \mathrm{C}$ to match the treatment of capped RNAs. The same RNA that was used in the

172 vaccinia capping reaction was directly compared to the post-cap RNA.

174 Generating P. falciparum in vitro translation lysates

176 hematocrit in RPMIc medium (RPMI 1640 media supplemented with 0.25\% Albumax II

177 (GIBCOLife Technologies), $2 \mathrm{~g} / \mathrm{L}$ sodium bicarbonate, $0.1 \mathrm{mM}$ hypoxanthine, $25 \mathrm{mM}$ HEPES (pH 
1787.4 ), and $50 \mu \mathrm{g} / \mathrm{L}$ gentamicin), at $37^{\circ} \mathrm{C}, 5 \% \mathrm{O} 2$, and $5 \% \mathrm{CO} 2$. Cultures were maintained at $2-5 \%$

179 parasitemia.

In depth step-by-step protocols for lysate generation have been previously published

182 [5]. In summary, cultures were synchronized twice using 5\% sorbitol six hours apart. Once

183 cultures recovered to $10 \%$ parasitemia, they were used to seed two 500mL hyperflasks (Corning

184 10031). When cultures reached the late trophozoite stage at 10-20\% parasitemia, the cultures

185 were centrifuged for $5 \mathrm{~min}$ at $1500 \mathrm{~g}$ at room temperature with no break, the supernatant was

186 removed, and $0.025-0.05 \%$ final saponin (exact amount determined by optimization of each

187 batch of saponin) in Buffer $\mathrm{A}$ (20 mM HEPES pH8.0, 2mM Mg(OAc) 2, 120mM KOAc) was added.

188 Saponin lysed cultures were centrifuged at $4^{\circ} \mathrm{C}$ at $10,000 \mathrm{~g}$ for $10 \mathrm{~min}$ in a Beckman Coulter

189 J26XPI. Pellets were washed twice with buffer A with centrifuging between each wash and then

190 were re-suspended in an equal volume to the pellet of BufferB2 (20 mM HEPES pH8.0,100 mM

$191 \mathrm{KOAc}, 0.75 \mathrm{mMMg}(\mathrm{OAC})_{2}, 2 \mathrm{mMDTT}, 20 \%$ glycerol,1XEDTA-free protease inhibitor cocktail

192 (Roche)), flash frozen, and stored in $-80^{\circ} \mathrm{C}$. Frozen pellets were then thawed at $4^{\circ} \mathrm{C}$ and lysed by

193 passing them through a cell homogenizer containing a $4 \mu \mathrm{m}$-clearance ball bearing (Isobiotec,

194 Germany) 20 times by hand or using a custom build machine [31]. The whole-cell lysate was

195 then centrifuged at $4^{\circ} \mathrm{C}$ at $16,000 \mathrm{~g}$ for $10 \mathrm{~min}$ and the supernatant was flash frozen and stored

196 at $-80^{\circ} \mathrm{C}$. The experiments performed here used a pool of lysates from multiple different

197 harvests that were each individually tested for a minimal activity of $10^{4}$ using a high expression

198 RNA containing NanoLuc (A[WT]) and the Promega Nano-Glo Luciferase assay system (Promega

$199 \mathrm{~N} 1110)$. Pooled lysates were then optimized for the needed amount of $\mathrm{Mg}(\mathrm{OAc})_{2}$ and an 
optimal incubation time at $37^{\circ} \mathrm{C}$, in this case $3 \mathrm{mM}$ final concentration $\mathrm{Mg}(\mathrm{OAc})_{2}$ and 57

201 minutes.

202

203

Generating $\mathrm{K} 562$ in vitro translation lysates

204

K562 suspension cells were cultured in RPMI 1640 media supplemented with $10 \%$ fetal bovine serum, 10mM Hepes (pH 7.2-7.5), and 0.5mg/mL Penicillin-Streptomycin-Glutamine. room temperature and the supernatant was removed. Pellets were washed twice with buffer $A$ with centrifuging at $1500 \mathrm{~g}$ at $4^{\circ} \mathrm{C}$ between each wash. Finally, pellets were re-suspended in an

211 equal volume of Buffer B2 and flash frozen in liquid nitrogen. Cell lysates were generated from

212 the frozen pellets using the same methodology as P. falciparum lysates, but with the cell

213 homogenizer containing a $12 \mu \mathrm{m}$-clearance. Lysates that produced over $10^{4}$ luminescence units

214 sing a high expression RNA containing NanoLuc Luciferase (A[WT]) and the Promega Nano-Glo

215 Luciferase assay system (Promega N1110) in preliminary tests were pooled and optimized for

216 the needed amount of $\mathrm{Mg}(\mathrm{OAc})_{2}$ and incubation time using $\mathrm{A}[\mathrm{WT}] \mathrm{mRNA}$, in this case $1.5 \mathrm{mM}$

217 final concentration $\mathrm{Mg}(\mathrm{OAc})_{2}$ and 12 minutes.

\section{In vitro translation protocol} In vitro translation reactions for $P$. falciparum and K562 lysates were set up identically. 
222 of 3.5uL lysate with 0.5uL 100uM complete amino acid mix (Promega L4461) and 1uL 10x

223 translation buffer (20mM Hepes pH 8, 75mM KoAc, 2mM DTT, 5mM ATP, 1mM GTP 200mM

224 creatine phosphate, $2 \mathrm{ug} / \mathrm{ul}$ Creatine kinase, and the pre-determined concentration for each

225 lysate pool of $\mathrm{Mg}(\mathrm{OAc})$ ) was added to each well. Reactions are then incubated for the pre-

226 determined amount of time at $37^{\circ} \mathrm{C}$, then placed on ice to stop the reactions. $8 \mathrm{uL}$ of reaction

227 was mixed with $8 \mathrm{uL}$ of Nano-Glo buffer/substrate mix following the Nano-Glo Luciferase Assay

228 System (Promega N1110) instructions. Luminescence was measured on a Promega GloMax

229 Plate Reader (Promega TM297) with a 6 second integration time.

231 Analysis

232

\section{Experimental TES}

All experiments were performed three separate times in triplicate, for a total of 9 values

236 per mRNA tested (except for the capped and uncapped experiment which was done 3-4 times

237 in duplicate). For each separate experiment, new mRNA was generated and capped. For the

238 figures, each value was normalized to the mean of the triplicates from each separate run. All

239 raw values and normalized values can be found in Additional File 4. The fold differences were

$240 \log _{2}$ transformed and then used to calculate the mean and SEM. Graphs for the figures were

241 made using a custom Python/Postscript script (Additional File 5).

243 Predicted activity of multiple uAUGs 
The percent repression of each UAUG individually was calculated by determining the percent of $\mathrm{R}[\Delta 1: \Delta 2: \Delta 3: \Delta 4]$ (the "maximum signal"). For the predictions, the percent repression

246 of each UAUG in the model were multiplied together.

Predicted Secondary Structures

To evaluate for secondary structure, the $\Delta G$ of 30 nucleotide stretches of the $5^{\prime}$ UTR

250 tiled with a 5 nucleotide separation was generated using RNAfold [32]. The predicted $\Delta \mathrm{G}$ were

251 then plotted using GraphPad Prism Software.

253 Results

254 Identifying putative cis-acting elements within the 5' UTRs of $\boldsymbol{P}$. falciparum that differ

To identify putative cis-acting sequences that regulate TE in P. falciparum, the ribosome

257 profiling and mRNA sequencing data generated by Caro and Ahyong et. al. [18] was re-analyzed

258 by comparing the $5^{\prime}$ UTR sequences of genes in the bottom $10 \%$ and top $10 \%$ of TEs during the

259 late trophozoite stage (Figure 1A). Features within the $5^{\prime}$ UTRs were quantified, and the

260 distributions from each set were compared. While the distributions of 5' UTR length were not

261 statistically distinct (K.S. test $p=0.10$ Supplemental Figure $1 \mathrm{~A}$ ), the distributions of uAUG

262 frequency differed significantly and appeared distinctly separated when normalized to 5' UTR

263 length with lower TE genes tending to contain more UAUGs (K.S. test $p=3.36^{*} 10^{-9}$ and

$264 p=1.1 * 10^{-21}$ respectively) (Supplemental Figure 1B, Figure $1 B$ ). This trend appeared to be most

265 distinct closest to the protein coding region (Figure $1 \mathrm{C}$ ). 
268 low and high TE (K.S. test $p=2.24 * 10^{-5}$ ) (Figure 1D). The positional effect followed a similar trend

269 with repressed genes on average having a higher GC content, especially near the translational

270 start site (Figure 1E). Together, this retrospective bioinformatic analysis suggested that these

271 two features should be further investigated for their role in influencing TE with particular

272 attention placed on the sequence region proximal to the translation start site.

274 Evaluating $P$. falciparum and human $\mathrm{K} 562$ in vitro translation assays for measuring the effect

275 of 5' UTRs on TE

To investigate the role of cis-acting elements within 5' UTRs, an in vitro translation assay

277 previously developed for identifying translation inhibitors against $P$. falciparum $[5,10]$ was

278 adapted using both $P$. falciparum W2 and $H$. sapiens $\mathrm{K} 562$ cellular extracts. To validate and

279 optimize the platform for this purpose, two mRNAs transcribed in late trophozoites with

280 significantly different TEs were identified, PF3D7_1411400 (a plastid replication-repair enzyme)

281 representing a translationally repressed mRNA from the bottom $10 \%$ of TEs and

282 PF3D7_1428300 (a proliferation-associated protein) representing a high translation mRNA from

283 the top $10 \%$ of TEs. These two genes were chosen for their relatively similar 5' UTR lengths and

284 other properties (Figure 2A and B). The full length $5^{\prime}$ UTRs of both genes (Figure 2A) were

285 cloned into a reporter construct driving expression of a luciferase enzyme and were evaluated

286 for their effect on TE. 
The 5' UTR of PF3D7_1411400 is 730 nucleotides long, contains 15 uAUGs (13 form

291 PF3D7_1428300 is 775 nucleotides long, contains 10 uAUGs (all of which form uORFs), and is

$2929.3 \%$ GC (Figure 2B). The abundance for the RNA was measured to be 522.93 reads per million

293 and the $\log _{2}(T E)$ was 1.75. Thus, the TE of the active gene is 12.2 -fold higher than that of the

294 repressed gene by ribosome profiling. In the $P$. falciparum in vitro translation assay, which

295 effectively removes any influence from differential expression levels, the signal produced by the

296 activating 5' UTR was 24.5-fold higher than the signal from the repressive 5' UTR (Figure 2B). In

297 the K562 in vitro translation assay, the 5' UTR from the active gene also out-performed that of

298 the repressed gene by 5.3-fold (Figure 2C). Both in vitro translation assays recapitulated the

299 difference in TE that was observed in vivo, albeit with different absolute magnitudes.

As noted above, the 5' UTR analysis of the ribosome profiling data suggested that

302 differences between high and low TE 5' UTRs appeared to be exaggerated closer to the

303 translation start site. To investigate this while reducing the search space for cis-acting elements,

304 each of the $5^{\prime}$ UTRs was progressively trimmed from the $5^{\prime}$ end (Figure 2C). In P. falciparum

305 lysates, shortening the activating 5' UTR to 549 nucleotides increased translation 4.2-fold, and

306 reducing the UTR to 130 nucleotides further increased translation 1.9-fold, for a 7.9-fold total

307 increase. Reducing the repressive 5' UTR to 339 nucleotides similarly increased translation 3.15-

308 fold, but further reduction to 130 nucleotides resulted in no additional increases in $P$.

309 falciparum. Similarly, in human K562 lysates, trimming of the 5' UTRs resulted in an overall 
310 increase in translation for both 5' UTRs and increased the TE differential between the two

311 (Figure 2B).

While trimming both $5^{\prime}$ UTRs increased their respective translation, the differential

314 between the activating and repressive UTRs was magnified. At 130 nucleotides, the activating 5'

315 UTR outperformed the repressive 5' UTR by 64-fold (Figure 2B), which had the added benefit of

316 increasing the dynamic range between constructs. Hence forth, the minimal 130 nucleotide

317 sequences were used as the platform for further dissection of cis-acting sequences and all

318 subsequent 5' UTRs evaluated were 130 nucleotides. The activating 130 nucleotide 5' UTR

319 derived from PF3D7_1428300 is denoted as A[WT] and the repressive 130 nucleotide 5' UTR

320 from PF3D7_1411400 is denoted as R[WT]. Reflective of the distinct distributions in uAUG

321 abundance and GC abundance, R[WT] is $16.9 \%$ GC and contains four uAUGs, numbered 1-4

322 based on distance from the translation start site. UAUGs 1 and 2 do not form uORFs and are in

323 the +1-frame relative to the reporter gene starting at -13 and -22 nucleotides, while uAUGs 3

324 and 4 both form uORFs at -66 and -101 nucleotides. A[WT] is $7.7 \%$ GC and contains no

325 upstream "AUG"s (Figure 2D).

$326 \quad$ All the RNAs used herein were capped using Vaccinia Capping Enzyme (NEB M2080S). To

327 verify that both lysates were sensitive to capping, capped and uncapped versions of the full

328 length 5' UTRs and the 130 nucleotide 5' UTRs were compared (Supplemental Figure 2). Both

329 lysates were sensitive to capping, with capped RNAs generally generating more luminescence

330 (up to a 21.7-fold increase in P. falciparum and 7.1 in K562 with full length 1429300), especially

331 in P. falciparum lysates. Additionally, in K562 lysates, uncapped RNAs with the full length 5' 
332 UTRs generated a more variable signal than capped RNAs. To promote scanning initiation,

333 increase luminescence signal, and reduce noise, all further experiments in this study utilized

334 capped RNA.

335 Measurement of both independent and combined effects of uAUGs on translational

336 repression

337 The combined effect of the four uAUGs in R[WT] was first evaluated by mutating all four

338 to "AUC", denoted $\mathrm{R}[\Delta 1 \Delta 2 \Delta 3 \Delta 4]$. Conversion of all four alleviated repression by over $1000 \%$ in

339 P. falciparum, and $337 \%$ in human lysates (Figure 3A). If each uAUG equally contributed toward

340 repression, the expected result of maintaining any single UAUG would be a consistent relief

341 from repression relative to R[WT]. However, individually maintaining each of the four uAUGs

342 yielded significantly different degrees of translation (Figure 3B), ranging from a modest 2-fold

343 increase with UAUG-3 alone $(\mathrm{R}[\Delta 1 \Delta 2 \Delta 4])$ to a nearly 10 -fold increase with uAUG-1 alone

$344(\mathrm{R}[\Delta 2 \Delta 3 \Delta 4])$, indicating unequal contributions towards the overall level of repression. For $\mathrm{K} 562$

345 extracts, the results were similar, although uAUG-2 alone ( $R[\Delta 1 \Delta 3 \Delta 4])$ was the most repressive

346 of the set, being even more so than the wild-type construct. Since UAUG-4 forms a uORF whose

347 stop site overlaps with uAUG-3 and was eliminated by making uAUG-3 into "AUC", uAUG-4 with

348 a restored $u$ ORF was also evaluated ( $\mathrm{R}[\Delta 1 \Delta 2 \Delta 3-\mathrm{uORF}$ restored]). With the uORF restored,

349 uAUG-4 confers minimal or no translational repression. These data demonstrate that each of

350 the individual uAUGs in isolation possess differing repressive activities with respect to

351 translation. 
To further evaluate the repressive effects of uAUGs in a novel context, the four uAUGs

354 from R[WT] were placed into A[WT] at the matching positions (Supplemental Figure 3). As

355 expected, in P. falciparum, when all four uAUGs were present $A[+1:+2:+3:+4]$, translation was

356 repressed, 2.9-fold. Additionally, each uAUG individually repressed translation between 1.5-fold

357 and 2.9-fold when the other positions were mutated to "AUC (Supplemental Figure 3). The

358 results in $\mathrm{K} 562$ followed the same trends as $P$. falciparum.

To explore potential interactions between uAUGs, pairwise combinations of the uAUGs

361 in R[WT] were evaluated (Figure 3C). If uAUGs possess independent repressive potentials that

362 do not affect each other, the repression by any two uAUGs would be the product of their

363 respective potentials. For example, the two furthest uAUGs, uAUG-1 and uAUG-4, yielded 37\%

364 and $73 \%$ of the maximum translation of the derepressed construct $\mathrm{R}[\Delta 1 \Delta 2 \Delta 3 \Delta 4]$ in $P$.

365 falciparum lysates. Thus, if acting independently, the predicted yield for a 5' UTR containing

366 both uAUGs would equal $0.37 * 0.73$, or $27 \%$, of the maximum signal. The measured signal for

367 this combination ( $\mathrm{R}[\Delta 2 \Delta 3])$ was extremely close to the predicted value, $28.6 \%$, suggesting that

368 these two elements act independently and proportionately on translation. Evaluation of the

369 remaining pairs of UAUGs revealed some notable combinations that likely highlight interacting

370 pairs (Supplemental Figure 4). Of note, the predicted combination of uAUG-3 and uAUG-4

$371(\mathrm{R}[\Delta 1 \Delta 2])$ in $P$. falciparum underestimates the measured amount of translation (11\% predicted

372 versus $19 \%$ measured), suggesting an interaction between uAUG-4 and uAUG-3, which, as

373 noted previously, marks the end of the uORF formed by uAUG-4. For K562 lysates, constructs 
containing uAUG-2 differ most from their predicted values, indicating this element may be

375 uniquely sensitive to the presence of the other uAUGs.

Having examined all pair-wise combinations of the four uAUGs, each three-way

381 independently confer varying levels of repression; however, multiple uAUGs may combine to

382 produce a concerted effect that was not predicted by their individual contributions.

\section{Investigating the effect of position and termination status on uAUG repression} relative to the translation start site, and their termination status. Previous work describing the

387 Kozak context for $P$. falciparum suggests a string of adenosine bases preceding the start site is 388 most commonly observed $[28,33]$. To assess the effects of uAUG positionality while maintaining 389 a common Kozak, a cassette comprised of the -3 to +9 sequence from uAUG-3 was individually 390 placed at five equally spaced positions within $\mathrm{R}[\Delta 1 \Delta 2 \Delta 3 \Delta 4]$ beginning at -14 nucleotides from

391 the reporter protein coding region (Figure 4). All cassettes were inserted in the +2 frame such

392 that if translation initiated at these sites, no reporter should be translated in-frame. Two

393 versions of the cassette were created, one maintaining the termination with a stop codon at

394 the end of the cassette and one without (Figure 4A/B). For the five constructs containing a non-

395 terminating UAUG, all potential stop sites proceeding the protein coding region in-frame with 
the $5^{\prime}$ most cassette were eliminated and the effect of these mutations alone in the presence of uAUG-3 $\left(\mathrm{R}[\Delta 1 \Delta 2 \Delta 4]^{*}\right)$ were evaluated (Supplemental Figure 5A). cassette placements resulted in repression comparable to $\mathrm{R}[\Delta 1 \Delta 2 \Delta 4]$ (Figure $4 \mathrm{C}$ ). Of note, the cassettes placed nearest to the $5^{\prime}$ cap had little effect on translation in either $P$. falciparum or K562 lysates (1.2-fold and 1.3-fold repression respectively). For $P$. falciparum, unlike the relative consistency of repression produced by uORF placement, the UAUG equivalent yielded a trend in repression. As the UAUG moved closer to the translation start site the repressive

405 strength increased until maximum repression was achieved when the cassette was placed -41

406 nucleotides from the translation start site (Figure 4C). In comparison, K562 lysates also yielded 407 peak repression at the -41 position, but the pattern of repression induced by both the uORF

408 and uAUG cassettes were more similar to each other and the trend observed for uAUG

409 cassettes in P. falciparum. These experiments indicate that in both P. falciparum and K562

410 lysates, the position of uAUGs contributes in part to downstream repression, however,

411 termination status may also impact this effect, at least in the case of $P$. falciparum.

\section{Evaluating the effect of GC content on TE}

415 content, especially within the intergenic regions that are $\sim 90 \%$ AT [34]. As noted in Figure 1D

416 and $1 \mathrm{E}$, there is a significant difference in the distributions of GC content between the 5' UTRs

417 of genes with high and low TE with repressed genes exhibiting a higher GC bias. These 
418 differences are evident within $A[W T]$ and $R[W T]$, which possess $7.7 \%$ GC, and $16.9 \%$ GC

419 respectively. This $\mathrm{GC}$ bias is intensified in the 60 nucleotides closest to the translation start with

$420 \mathrm{~A}[\mathrm{WT}]$ containing only $1.7 \% \mathrm{GC}$ and $\mathrm{R}[\mathrm{WT}]$ containing $15 \% \mathrm{GC}$ (Figure 2D). To investigate the

421 impact of GC content in the context of these two constructs, substitutions were systematically

422 introduced into the proximal region of $A[W T]$ to increase the GC content from $1.7 \%$ to a

423 maximum of $30 \%$ GC (Figure 5A). Substitutions were maintained between constructs, no

424 upstream "AUG"s were introduced, and significant secondary structures was avoided

425 (Supplementary Figure 5). In P. falciparum lysates, between $1.7 \%$ and $20 \%$ GC there was no

426 change in TE while at 30\% GC translation was repressed 1.5-fold (Figure 5A). The repressive

427 effect of the high GC content was 1.3-fold in human K562 lysates.

428

429 The converse experiment of reducing the GC content of R[WT] was also carried out. The

$430 \mathrm{GC}$ content in the last 60 nucleotides of $\mathrm{R}[\Delta 1 \Delta 2 \Delta 3 \Delta 4]$ was reduced to $5 \%$ by eliminating all GC

431 content between 4 and 60 nucleotides from the translation start site and to $0 \%$ by removing all

432 GC (Figure 5B). A maximum translation increase of approximately 2-fold was observed relative 433 to $\mathrm{R}[\Delta 1 \Delta 2 \Delta 3 \Delta 4]$, indicating a modest but measurable impact in this context. These results were 434 mirrored in K562 lysates (Figure 5B). Together, the result of manipulating the GC content of the 435 last 60 nucleotides of the 5' UTR suggests that the impact on translation to be subtle, but 436 sensitive to the overall context. 
In addition to the study of specific elements predicted to impact TE, a series of systematic sequence swaps were investigated, in which regions from both the 5' and 3' end of

$441 \mathrm{R}[\mathrm{WT}]$ and $\mathrm{A}[\mathrm{WT}]$ were exchanged. Beginning with the $3^{\prime}$ end of the $5^{\prime}$ UTR, 20, 40, and 60

442 nucleotides were exchanged between R[WT] and A[WT] (Figure 6A and 6B). In the case of

$443 \mathrm{~A}[\mathrm{WT}]$, introducing more sequence from $\mathrm{R}[\mathrm{WT}]$ severely impacted TE. While some of this

444 impact was anticipated due to the introduction of UAUG-1 and UAUG-2, additional decreases in

445 translation were observed with sequence beyond these elements ( $A[60$ nt 3' R] ). Furthermore,

446 the added impact beyond the introduction of UAUGs was observed only with $P$. falciparum

447 Iysates. For the converse experiments, exchange of sequence from A[WT] into R[WT] at the 3'

448 end resulted in increased translation (11.7-fold). This increase in translation was in part

449 expected due to the elimination of UAUG-1 and UAUG-2, however the magnitude of the effect is

450 greater than predicted from the experiments shown in Figure 3C. The effect in human K562

451 lysates was markedly less with a maximum difference of 1.4-fold.

452 Sequence exchanges at the 5' end were similarly carried out using 10, 20, and 30

453 nucleotide swaps between $\mathrm{A}[\mathrm{WT}]$ and $\mathrm{R}[\Delta 1: \Delta 2: \Delta 3: \Delta 4]$. The latter construct was chosen over

$454 \mathrm{R}[\mathrm{WT}]$ to assess the impact in the absence of uAUGs. For $P$. falciparum, exchanging the first 10

455 nucleotides of $\mathrm{R}[\Delta 1: \Delta 2: \Delta 3: \Delta 4]$ into $\mathrm{A}[\mathrm{WT}]$ repressed translation 2.6-fold, with a final 3.7-fold

456 repression exchanging 30 nucleotides. (Figure 6C). In contrast, exchanging the first 10 and 20

457 nucleotides of $A[W T]$ into $R[\Delta 1: \Delta 2: \Delta 3: \Delta 4]$ activated translation up to 1.9-fold while exchanging

45830 nucleotides activated translation 3.5-fold. Note that the level of translation achieved in this

459 latter construct matches the output of A[WT], demonstrating that in the absence of uAUGs, 
460 exchanging the sequence elements within the first 30 nucleotides of the $5^{\prime}$ end of the 5' UTR

461 was sufficient to render $\mathrm{A}[\mathrm{WT}]$ and $\mathrm{R}[\Delta 1: \Delta 2: \Delta 3: \Delta 4]$ approximately equivalent (Figure $6 \mathrm{D}$ ).

463 Discussion

Among eukaryotes, P. falciparum presents several distinct features that bear upon translation. First, the AT-rich genome contains frequent poly-adenosine stretches that alone

466 necessitates unique adaptions of the translational machinery to prevent ribosome stalling or

467 frameshifting [35,36]. Additionally, there are a limited number of ribosomal RNA copies with in

468 the genome, each with stage specific expression [37,38]. The transcriptome also features

469 unusually long 5' UTRs, the longest in late trophozoites being a remarkable 8229 nucleotides

470 (PF3D7_1139300). Despite these features, previous studies suggest that $P$. falciparum initiates

471 translation in a cap-dependent manner similarly to other eukaryotes $[39,40]$.

While the central initiation factors required for cap-binding have been bioinformatically

474 identified and many of the essential interactions have been validated, questions remain around

475 how these factors regulate translation initiation given $P$. falciparum's unique 5' UTR features

$476[26,41]$. Additionally, ribosome profiling has demonstrated that translation is an integral point

477 of regulation for model eukaryotes [42,43], but for $P$. falciparum it reveals that less than $10 \%$ of

478 transcripts are translationally regulated. Directly evaluating how these unusual mRNA features

479 function in P. falciparum could reveal unique mechanisms that would be powerful therapeutic 480 targets. 
A re-analysis of ribosome profiling data highlights two important features that differ

483 between mRNAs at the top and bottom of the TE range. As shown in Figure 1, the presence of

484 uAUGs and GC content are significantly different between highly translated and poorly

485 translated mRNAs, a difference that appears exacerbated by proximity to the protein coding

486 region. To explore and dissect the role of these features, two representative 5' UTRs were

487 chosen from the top and bottom deciles, the 5' UTRs of PF3D7_1411400 and PF3D7_1428300.

488 The differences in TE driven by these two 5' UTRs were faithfully recapitulated using in vitro

489 translation extracts generated from late trophozoites of $P$. falciparum W2 strain (Figure 2) and

490 human K562 cells. Surprisingly, these differences were maintained when using only the

491 proximal 130 nucleotides from each 5' UTR, with A[WT] derived from PF3D7_1428300 and

492 R[WT] from PF3D7_1411400. These two 130-nucleotide 5' UTRs provided an ideal platform to

493 evaluate the effects of UAUGs and GC content.

uAUGs have long been appreciated as translational regulatory elements, and work by

496 Marilyn Kozak demonstrated their repressive abilities in the early 1980s [20]. However, it

497 remains difficult to predict the individual or joint repressive activities of uAUGs from sequence

498 context alone, especially for non-model organisms. Additionally, it is unusually to have uAUGs

499 so abundant throughout the transcriptome. Here, a reductionist approach was used to

500 individually assess the repressive potential of each uAUG within R[WT] in isolation, and in

501 combination (Figure 3). For many pairs, such as UAUG-1 and uAUG-4, the combined activity

502 directly reflected a combination of each UAUG's repressive strength. For others, like uAUG-3

503 and UAUG-4, it was revealed that the combined effect of two uAUGs could be reduced by their 
504 interaction. Since UAUG-3 is itself the in-frame stop site for UAUG-4, it reasonable to assume

505 that the termination of UAUG-4 may interfere with initiation events at uAUG-3. These

506 interactions make it difficult to predict the impact of multiple uAUGs without direct

507 measurements as performed here.

508

509

The sequence context surround an "AUG" is essential for determining the rate of

510 initiation at that site $[11,44,45]$, however, additional elements may affect the regulatory activity

511 of an uAUG. Here, two possible modifiers were examined in detail, namely, the position of

512 uAUGs relative to the protein coding region, and whether it forms a uORF (Figure 4). Both the

513 position and termination status affect translation with the most dramatic result arising when

514 the UAUG is positioned furthest from the protein coding region, only 11 nucleotides from the $5^{\prime}$

515 cap. At this distance neither the open UAUG nor the uORF repressed translation. One caveat of

516 this study is that only one putative uORF was assessed. It is likely that the length and

517 composition of the uORF sequence itself may modify the overall impact.

519 Along with uAUG frequency, bioinformatic analysis of the 5' UTR sequences from $P$.

520 falciparum also reveals a statistically significant difference in GC content, with higher GC

521 content corresponding to lower TE. While higher GC content could correlate with higher

522 secondary structures, we wanted to evaluate if GC content alone could regulate translation.

523 Surprisingly, the results of manipulating GC content proximal to the protein coding region in the

524 context of only these two chosen UTRs yielded corresponding changes in the predicted

525 direction, albeit with small magnitudes when compared to the impact of uAUGs. In the active 
526 context, translation became repressed relative to A[WT] at $30 \%$ GC content within 60

527 nucleotides of the translational start. Within this 60-nucleotide region, only $31(1.4 \%)$ of the

5282088 5' UTRs from P. falciparum expressed in late trophozoites evaluated here are $30 \%$ GC or

529 above (Figure 1A). Thus, few genes would be predicted to be impacted by these shifts in GC

530 content alone. Eliminating GC content from the last 60 nucleotides of $R[\Delta 1: \Delta 2: \Delta 3: \Delta 4]$ resulted

531 in modest increases in TE (Figure 5B). In this case, of the $20885^{\prime}$ UTRs $273(13.1 \%$ ) are $5 \%$ or

532 below within this region and $16(0.8 \%)$ are $0 \%$.

533

534 Finally, to examine the effects of the sequences within A[WT] and R[WT]/

$535 \mathrm{R}[\Delta 1: \Delta 2: \Delta 3: \Delta 4]$ on translation, segments from the $5^{\prime}$ and 3' ends were progressively exchanged

536 between them (Figure 6). Sequence exchanges at the 3' end of the 5' UTR removed or

537 introduced uAUGs, which resulted in the expected increases or decreases in TE respectively. We

538 note that in each case, exchanged sequence beyond the UAUGs also impacted TE in $P$.

539 falciparum, suggesting additional context within these regions. Sequence exchanges at the 5'

540 end were more impactful than would have been predicted. Specifically, 30 nucleotides of

$541 \mathrm{~A}[\mathrm{WT}]$, when substituted into $\mathrm{R}[\Delta 1: \Delta 2: \Delta 3: \Delta 4]$, suggest a possible sequence with a role in

542 regulating the rate of translation initiation.

As an essential pathway throughout the parasite's life cycle, protein synthesis is an

545 attractive therapeutic target. However, since the mechanisms of eukaryotic translation are

546 highly conserved, potential therapeutics must cross the challenging bar of being highly specific

547 to $P$. falciparum. Here, in vitro translation was used to allow for direct comparison between $P$. 
548 falciparum and human to identify unique effects on TE. Despite the large evolutionary distance

549 between the two organisms, $P$. falciparum and $\mathrm{K} 562$ lysates yielded highly similar results in the

550 context of the two short model UTRs used here. For developing therapeutics targeting

551 translation initiation, avoiding host effects will be challenging, but in vitro translation can

552 continue to be a valuable tool to directly measure differences between Plasmodium and

553 humans [46].

Finally, this work continues the task of uncovering the complexity of 5' UTR cis-acting

556 regulatory elements and their impact on TE in eukaryotes. In vitro translation has previously

557 revealed the importance of the Kozak consensus sequence and uAUGs in model eukaryotes,

558 such as Saccharomyces cerevisiae and mammalian cultures [21,47-49], while higher throughput

559 selection and machine learning techniques have been used to probe the effect of $5^{\prime}$ UTR cis-

560 acting elements in these same systems $[24,50]$. However, working with non-model organisms

561 such as $P$. falciparum poses unique challenges, such that many of these techniques cannot be

562 readily utilized for comparative analysis. The highly reductionist approach taken here has the

563 benefit of allowing specific and systematic hypotheses to be tested, although it is clear that

564 higher throughput methods will be required to generalize these findings beyond these specific

565 examples.

566

\section{Conclusions}

568 Cis-acting features within the 5' UTRs of eukaryotes regulate the TE of a given gene.

569 While specific examples have previously been evaluated in model eukaryotes, $P$. falciparum 
570 possesses unusual 5' UTR characteristics, such as length, base content, and high uAUG

571 prevelence, that suggest cis-acting upstream elements play a significant role in tuning

572 translational efficiencies. Through extensive dissection of exemplar 5' UTRs from $P$. falciparum,

573 we measure the individual impacts of each putative element while comparing these same

574 constructs in human lysates. The impact of these elements was found to be surprisingly similar

575 in both systems. Since, unlike humans and most other studies eukaryotes, long 5' UTRs

576 featuring multitudes of uAUGs are common in P. falciparum, the precise configuration of these

577 elements may have evolved to tune translation levels in this organism where other post-

578 transcriptional regulatory mechanisms may be absent.

579

580 List of abbreviations

581 UAUG-upstream "AUG"

582 uORF- upstream open reading frame

583 5' UTR-5' untranslated region

584 TE-translational efficiency

\section{Declarations}

$586 \quad$ Ethics approval and consent to participate

$587 \quad$ Consent for publication

$588 \quad$ Availability of data and material

589 The datasets supporting the conclusions of this article are included within the

590 article in Supplemental File 4. The TES and 5' UTR sequences from that data used for

591 comparative analysis here can be found in Supplemental File 1. The previously published 
data from Caro, Ahyong et. al. [18] can be found at available at Dryad Digital Repository under a CCO Public Domain Dedication: http://dx.doi.org/10.5061/dryad.vb855.

There are no competing interests for any of the authors pertaining to this work.

Funding was provided by the Chan Zuckerberg Biohub.

Authors' Contributions

VEG and JLD conceived and designed this study. VEG performed and executed the experiments. RD maintained, harvested, and generated in vitro translation lysates for the K562 cells. VEG and JLD drafted and edited this manuscript. All authors read and approved the submitted manuscript. Acknowledgements

We would like to acknowledge the DeRisi lab's Team Malaria for advice, for valuable discussion and comments on the manuscript and Hanna Retallack, Jamin Lui, Madhura Raghavan, Sara Sunshine, Elze Rackaityte, and Caleigh Mandle-Brehm for their edits and commentary on the paper.

\section{References}

611 1. World Malaria Report 2019 [Internet]. The World Health Organization; 2019. Available from: 612 https://www.who.int/publications-detail/world-malaria-report-2019

613 2. Wicht KJ, Mok S, Fidock DA. Molecular Mechanisms of Drug Resistance in Plasmodium 614 falciparum Malaria. Annu Rev Microbiol. Annual Reviews; 2020;74:431-54. 
3. Mathieu LC, Cox H, Early AM, Mok S, Lazrek Y, Paquet J-C, et al. Local emergence in Amazonia of Plasmodium falciparum k13 C580Y mutants associated with in vitro artemisinin resistance. Soldati-Favre D, Cui L, editors. eLife. eLife Sciences Publications, Ltd; 2020;9:e51015.

4. Imwong M, Suwannasin K, Kunasol C, Sutawong K, Mayxay M, Rekol H, et al. The spread of artemisinin-resistant Plasmodium falciparum in the Greater Mekong subregion: a molecular epidemiology observational study. The Lancet Infectious Diseases. 2017;17:491-7.

622 5. Sheridan CM, Garcia VE, Ahyong V, DeRisi JL. The Plasmodium falciparum cytoplasmic 623 translation apparatus: a promising therapeutic target not yet exploited by clinically approved 624 anti-malarials. Malaria Journal. 2018;17:465.

625 6. Baragaña B, Hallyburton I, Lee MCS, Norcross NR, Grimaldi R, Otto TD, et al. A novel multiple-stage antimalarial agent that inhibits protein synthesis. Nature. 2015;522:315-20.

7. Saint-Léger A, Sinadinos C, Ribas de Pouplana L. The growing pipeline of natural aminoacylInhibition of the Plasmodium falciparum Lysyl-tRNA Synthetase by the Fungal Secondary Metabolite Cladosporin. Cell Host Microbe. 2012;11:654-63.

9. Zhou J, Huang Z, Zheng L, Hei Z, Wang Z, Yu B, et al. Inhibition of Plasmodium falciparum Lysyl-tRNA synthetase via an anaplastic lymphoma kinase inhibitor. Nucleic Acids Research. 2020;48:11566-76. Plasmodium falciparum specific translation inhibitors from the MMV Malaria Box using a high throughput in vitro translation screen. Malaria Journal. 2016;15:173. Mechanisms and Biological Targets. Cell. 2009;136:731-45.

12. Aylett CHS, Ban N. Eukaryotic aspects of translation initiation brought into focus. Philos

642 13. Lind C, Åqvist J. Principles of start codon recognition in eukaryotic translation initiation. 643 Nucleic Acids Res. 2016;44:8425-32.

644 14. Hinnebusch AG. Structural Insights into the Mechanism of Scanning and Start Codon 645 Recognition in Eukaryotic Translation Initiation. Trends Biochem Sci. 2017;42:589-611. 
16. Vilela C, McCarthy JEG. Regulation of fungal gene expression via short open reading frames in the mRNA 5'untranslated region. Mol Microbiol. 2003;49:859-67.

651 17. Calvo SE, Pagliarini DJ, Mootha VK. Upstream open reading frames cause widespread 652 reduction of protein expression and are polymorphic among humans. Proc Natl Acad Sci U S A. 653 2009;106:7507-12.

654 18. Caro F, Ahyong V, Betegon M, DeRisi JL. Genome-wide regulatory dynamics of translation 655 in the Plasmodium falciparum asexual blood stages. Gingeras TR, editor. eLife. eLife Sciences 656 Publications, Ltd; 2014;3:e04106.

657 19. Zhang H, Wang Y, Lu J. Function and Evolution of Upstream ORFs in Eukaryotes. Trends in 658 Biochemical Sciences. 2019;44:782-94.

659 20. Kozak M. Selection of initiation sites by eucaryotic ribosomes: effect of inserting AUG 660 triplets upstream from the coding sequence for preproinsulin. Nucleic Acids Res. 1984;12:387366193.

662 21. Alghoul F, Laure S, Eriani G, Martin F. Translation inhibitory elements from Hoxa3 and 663 Hoxa11 mRNAs use uORFs for translation inhibition. Sonenberg N, Manley JL, editors. eLife. 664 eLife Sciences Publications, Ltd; 2021;10:e66369.

665 22. Hinnebusch AG. Gene-specific translational control of the yeast GCN4 gene by 666 phosphorylation of eukaryotic initiation factor 2. Mol Microbiol. 1993;10:215-23.

667 23. Gunišová S, Beznosková P, Mohammad MP, Vlčková V, Valášek LS. In-depth analysis of 668 cis-determinants that either promote or inhibit reinitiation on GCN4 mRNA after translation of 669 its four short uORFs. RNA. 2016;22:542-58.

670 24. Cuperus JT, Groves B, Kuchina A, Rosenberg AB, Jojic N, Fields S, et al. Deep learning of 671 the regulatory grammar of yeast 5' untranslated regions from 500,000 random sequences.

672 Genome Res. 2017;27:2015-24.

673 25. Shaw PJ, Ponmee N, Karoonuthaisiri N, Kamchonwongpaisan S, Yuthavong Y.

674 Characterization of human malaria parasite Plasmodium falciparum eIF4E homologue and 675 mRNA 5' cap status. Mol Biochem Parasitol. 2007;155:146-55.

676 26. Tuteja R. Identification and bioinformatics characterization of translation initiation complex 677 eIF4F components and poly(A)-binding protein from Plasmodium falciparum. Commun Integr 678 Biol. 2009;2:245-60.

679 27. Bancells C, Deitsch KW. A molecular switch in the efficiency of translation reinitiation 680 controls expression of var2csa, a gene implicated in pregnancy-associated malaria. Molecular 681 Microbiology. 2013;90:472-88.

682 28. Kumar M, Srinivas V, Patankar S. Upstream AUGs and upstream ORFs can regulate the 683 downstream ORF in Plasmodium falciparum. Malar J. 2015;14:512. 
29. Horrocks P, Wong E, Russell K, Emes RD. Control of gene expression in Plasmodium falciparum - ten years on. Mol Biochem Parasitol. 2009;164:9-25.

30. Chappell L, Ross P, Orchard L, Russell TJ, Otto TD, Berriman M, et al. Refining the transcriptome of the human malaria parasite Plasmodium falciparum using amplification-free RNA-seq. BMC Genomics [Internet]. 2020 [cited 2021 Apr 19];21. Available from: https://www.ncbi.nlm.nih.gov/pmc/articles/PMC7278070/

31. Garcia VE, Liu J, DeRisi JL. Low-Cost Touchscreen Driven Programmable Dual Syringe Pump for Life Science Applications [Internet]. Bioengineering; 2018 Mar. Available from: http://biorxiv.org/lookup/doi/10.1101/288290

32. Lorenz R, Bernhart SH, Höner Zu Siederdissen C, Tafer H, Flamm C, Stadler PF, et al. ViennaRNA Package 2.0. Algorithms Mol Biol. 2011;6:26.

33. Saul A, Battistutta D. Analysis of the sequences flanking the translational start sites of Plasmodium falciparum. Molecular and Biochemical Parasitology. 1990;42:55-62.

34. Gardner MJ, Hall N, Fung E, White O, Berriman M, Hyman RW, et al. Genome sequence of the human malaria parasite Plasmodium falciparum. Nature. 2002;419:498-511.

35. Erath J, Djuranovic S, Djuranovic SP. Adaptation of Translational Machinery in Malaria Parasites to Accommodate Translation of Poly-Adenosine Stretches Throughout Its Life Cycle. Front Microbiol [Internet]. Frontiers; 2019 [cited 2021 Jul 27];0. Available from:

https://www.frontiersin.org/articles/10.3389/fmicb.2019.02823/full

36. Pavlovic Djuranovic S, Erath J, Andrews RJ, Bayguinov PO, Chung JJ, Chalker DL, et al. Plasmodium falciparum translational machinery condones polyadenosine repeats. Sonenberg N, Manley JL, editors. eLife. eLife Sciences Publications, Ltd; 2020;9:e57799.

37. Waters AP, Syin C, McCutchan TF. Developmental regulation of stage-specific ribosome populations in Plasmodium. Nature. 1989;342:438-40.

38. Li J, Gutell RR, Damberger SH, Wirtz RA, Kissinger JC, Rogers MJ, et al. Regulation and trafficking of three distinct $18 \mathrm{~S}$ ribosomal RNAs during development of the malaria parasite11Edited by D. E. Draper. Journal of Molecular Biology. 1997;269:203-13.

39. Kaur C, Kumar M, Patankar S. Messenger RNAs with large numbers of upstream open reading frames are translated via leaky scanning and reinitiation in the asexual stages of Plasmodium falciparum. Parasitology. Cambridge University Press; 2020;147:1100-13.

\section{Amulic B, Salanti A, Lavstsen T, Nielsen MA, Deitsch KW. An Upstream Open Reading} Frame Controls Translation of var2csa, a Gene Implicated in Placental Malaria. PLOS Pathogens. Public Library of Science; 2009;5:e1000256.

41. Vembar SS, Droll D, Scherf A. Translational regulation in blood stages of the malaria parasite Plasmodium spp.: systems $\square$ wide studies pave the way. Wiley Interdiscip Rev RNA. 2016;7:772-92. 

Vivo of Translation with Nucleotide Resolution Using Ribosome Profiling. Science. American

722 Association for the Advancement of Science; 2009;324:218-23.

723 43. Brar GA, Weissman JS. Ribosome profiling reveals the what, when, where, and how of 724 protein synthesis. Nat Rev Mol Cell Biol. 2015;16:651-64.

725 44. Hinnebusch AG, Lorsch JR. The Mechanism of Eukaryotic Translation Initiation: New 726 Insights and Challenges. Cold Spring Harb Perspect Biol. 2012;4:a011544.

727 45. Maag D, Algire MA, Lorsch JR. Communication between Eukaryotic Translation Initiation 728 Factors 5 and 1 A within the Ribosomal Pre-initiation Complex Plays a Role in Start Site 729 Selection. Journal of Molecular Biology. 2006;356:724-37.

730 46. Ahyong V, Sheridan CM, Leon KE, Witchley JN, Diep J, DeRisi JL. Identification of Plasmodium falciparum specific translation inhibitors from the MMV Malaria Box using a high throughput in vitro translation screen. Malaria Journal. 2016;15:173.

733 47. Kozak M. Initiation of translation in prokaryotes and eukaryotes. Gene. 1999;234:187-208.

734 48. Kozak M. Features in the $5^{\prime}$ non-coding sequences of rabbit $\alpha$ and $\beta$-globin mRNAs that 735 affect translational efficiency. Journal of Molecular Biology. 1994;235:95-110.

736 49. Kozak M. Evaluation of the fidelity of initiation of translation in reticulocyte lysates from 737 commercial sources. Nucleic Acids Res. 1990;18:2828.

738 50. Karollus A, Avsec Ž, Gagneur J. Predicting mean ribosome load for 5'UTR of any length 739 using deep learning. PLOS Computational Biology. Public Library of Science;

$740 \quad 2021 ; 17: \mathrm{e} 1008982$.

Figure Legends

743 Figure 1: Comparison of features within the $5^{\prime}$ UTRs of genes in the bottom $10 \%(n=209)$ and

744 top $10 \%(n=209)$ of TEs in the late trophozoite stage using data from Caro and Ahyong et. al.

$7452014[18]$.

746 a) A histogram of the $\log _{2}(T E) s$ of genes expressed in the late trophozoite stage included in

747 subsequent analysis. The vertical dotted lines indicate the bottom $10 \%$ (yellow) and top $10 \%$

748 (blue) of 5' UTRs. 
749 b) The number of uAUGs normalized to the length of the 5' UTRs in the bottom 10\% (yellow)

750 and top $10 \%$ (blue) of TEs in the late trophozoite stage. The two distributions are statistically

751 distinct, KS test-statistic 0.47 , p-value $1.4 * 10^{-21}$.

752 c) The average number of UAUGs in the 5' UTRs within a 130-nucleotide window sliding by 5

753 nucleotides up to 1000 nucleotides of the bottom $10 \%$ (yellow) and top $10 \%$ blue.

754 d) The distribution of GC content in the bottom $10 \%$ (yellow) and top $10 \%$ (blue) of TEs in the

755 late trophozoite stage are statistically distinct, KS test statistic comparison of the two: $0.23 \mathrm{p}$ -

756 value $2.24 * 10^{-5}$

757 e) The average GC content within a 130-nucleotides sliding window moving 5 nucleotides up to

7581000 nucleotides from the translation start site. Bottom 10\% (yellow) and top 10\% blue.

759

760 Supplemental Figure 1: Further comparison of 5' UTR features of genes in the bottom $10 \%$ and

761 top $10 \%$ of TEs in the late trophozoite stage using data from Caro and Ahyong et. al. 2014 [18].

762 a) Distributions of the 5' UTR lengths from genes with high (blue) or low (yellow) TE. KS test

763 statistic comparison of the two: $0.12 p$-value 0.1

764 b) Distributions of the total number of uAUGs in the $5^{\prime}$ UTRs from genes with high (blue) or low

765 (yellow) TE. KS test statistic comparison of the two: $0.31 \mathrm{p}$-value $1.5^{*} 10^{-5}$.

766

767 Figure 2: 130 nucleotides of the 5' UTR from a translationally active (PF3D7_1428300) and

768 repressed gene (PF3D7_1411400) were sufficient to drive distinct TE.

769 a) The diagramed sequence of the full length 5' UTRs from active PF3D7_1428300 and

770 repressed PF3D7_1411400. uAUGs are marked by green triangles with the different shades 
771 representing the three frames. The numbers between the two construct diagrams mark

772 distance from the protein coding region.

773 b) The lengths, uAUG count, uORF count, GC content, and translational efficiency (TE) for the

774 chosen 5' UTRs and genes obtained from the previously published ribosome profiling and

775 mRNA sequencing [18] with the raw luminescence signal (PF RLU) produced by these 5' UTRs

776 driving NanoLuc (Promega) expression using P. falciparum in vitro translation.

777 c) $\log _{10}$ (luminescence) from NanoLuc produced by $5^{\prime}$ UTRs of decreasing length in P. falciparum

778 lysates (red) and K562 lysates (grey). The different lengths were generated by shorting the 5'

779 UTRs from the 5' end.

780 d) Sequence comparison of the 130 nucleotides closest to the protein coding region of the $5^{\prime}$

781 UTRs from PF3D7_1411400 (R[WT]) and PF3D7_1428300 (A[WT]). The four uAUGs in R[WT] are

782 labeled with the green triangles. UAUGs without in-frame stops are followed by a dotted line

783 while uORF forming UAUGs are followed by a solid line with the stop is marked by a vertical

784 line. The four uAUGs are labeled 1-4 based on their distance from the protein coding start site.

786 Supplemental Figure 2: The raw luminescence signal from capped and uncapped RNAs in $P$.

787 falciparum and K562 in vitro translation.

788 Figure 3: Dissecting the effects of the four uAUGs in R[WT]. uAUGs were found to have a

789 generally repressive effect on TE that can be dependent on the presence each other. Graphed

790 for each is the average and SEM of $\log _{2}$ (each experimental value normalized to experimental

$791 \mathrm{R}[\mathrm{WT}]$ mean). The dotted line marks the average $\log _{2}(\mathrm{R}[\Delta 1 \Delta 2 \Delta 3 \Delta 4]$ normalized to $\mathrm{R}[\mathrm{WT}])$. For 
792 each figure, to the left is a diagram of the sequences using the same annotations as Figure $2 \mathrm{C}$.

793 To the right of the diagrams are the results for $P$. falciparum and human K562s.

794 a) The effect of removing all four UAUGs from R[WT].

795 b) The effect of retaining a single UAUG

796 c) The effect of removing each uAUG individually

797 d) The effect of removing two uAUGs in combination

799 Supplemental Figure 3: The 4 uAUGs from R[WT] exchanged into A[WT] at the same positions

800 showing that the repressive effect is conferrable to other contexts. Graphed for each is the

801 average and SEM of $\log _{2}$ (each experimental value normalized to the experimental average of $802 \mathrm{~A}[\mathrm{WT}])$.

804 Supplemental Figure 4: Predicted repressive effect of combinations of the UAUGs in R[WT]

805 based on their individual activities for a) P. falciparum and b) K562.

807 Figure 4: Effect of equally spaced and out of frame, non-terminated uAUGs or uORFs on TE

808 a) Sequence diagram of the two cassettes inserted into $R[\Delta 1: \Delta 2: \Delta 3: \Delta 4]$ at 5 different positions.

809 The green arrows mark the uAUGs, the solid line indicates the length of the uORF, and the

810 dotted line marks the sequence downstream of the non-terminated uAUG

811 b) The sequence diagrams to the left represent the $5^{\prime}$ UTRs containing the uORF cassette. To

812 the right the uORF cassette and the non-terminated cassette are presented side by side. The 
813 left set is from $P$. falciparum lysates while the right is from human. Graphed for each is the

814 average and SEM of $\log _{2}($ each experimental value/ experimental mean of $R[\Delta 1: \Delta 2: \Delta 3: \Delta 4])$.

816 Supplemental figure 5: To eliminate all downstream stop sites for moving the out of frame non-

817 terminated uAUG, 6-point mutations had to be added to the 5' UTR. R[ $\Delta 1: \Delta 2: \Delta 4]^{*}$ was made

818 with those point mutations to compare to $\mathrm{R}[\Delta 1: \Delta 2: \Delta 4]$. Graphed for each is the average and

819 SEM of $\log _{2}$ (each triplicate value/ average of $\mathrm{R}[\Delta 1: \Delta 2: \Delta 3: \Delta 4]$ experimental triplicates)

820

821 Figure 5: Evaluating the effect of GC content on translation

822 a) Increasing GC content in A[WT]. Graphed for each is the average and SEM of $\log _{2}$ (each

823 experimental value/ experimental mean of $A[W T])$

824 b) eliminating GC content in $\mathrm{R}[\Delta 1: \Delta 2: \Delta 3: \Delta 4]$. Graphed for each is the average and SEM of

$825 \log _{2}$ (each experimental value/ experimental mean of $\left.R[\Delta 1: \Delta 2: \Delta 3: \Delta 4]\right)$

826

827 Supplemental figure 6: The predicted free energy of the secondary structure with in a 30-

828 nucleotide sliding window moved by 1 nucleotide across the $5^{\prime}$ UTRs used to evaluate the effect

829 of GC content.

830 Figure 6: Evaluating the effects of the ends of the 5' UTRs on translation

831 a) Swapping the $3^{\prime}$ end of R[WT] into A[WT] Graphed for each is the average and SEM of

$832 \log _{2}$ (each experimental value/ mean experimental A[WT])

833 b) swapping the $3^{\prime}$ end of A[WT] into R[WT]. Graphed for each is the average and SEM of

$834 \log _{2}$ (each experimental value / mean experimental R[WT]) 
835 c) swapping the $5^{\prime}$ end of $\mathrm{R}[\Delta 1: \Delta 2: \Delta 3: \Delta 4]$ into A[WT]. Graphed for each is the average and SEM

836 of $\log _{2}$ (each experimental value / mean experimental of A[WT])

837 d) swapping the $5^{\prime}$ end of $A[W T]$ into R[WT]. Graphed for each is the average and SEM of

$838 \log _{2}$ (each experimental value / mean experimental $\left.R[\Delta 1: \Delta 2: \Delta 3: \Delta 4]\right)$.

$840 \quad$ Additional Files

841 Additional File 1: 5' UTR analyzed. The data on the 2088 genes used for the analysis in Figure 1

842 and Supplemental Figure 1, including the 5' UTR sequences used, .xIs.

843 Additional File 2: P16 sequence. The plasmid sequence for P16 used to generate new

844 constructs, . geneious.

845 Additional File 3: 5' UTR sequences. The sequences of all the 5' UTR sequences evaluated in this

846 manuscript, .fasta.

847 Additional File 4: Figure data. All the raw and processed data used to generate the figures in

848 this manuscript, .xls.

849 Additional File 5: Figure generating script. The script used to generate the figures within the

850 manuscript, .py. 
A

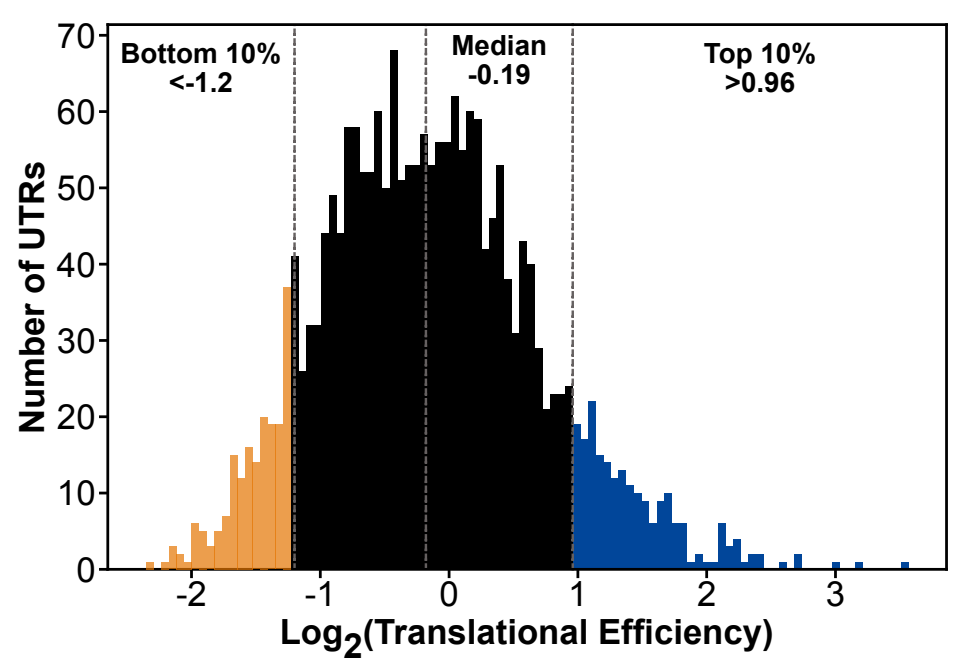

B

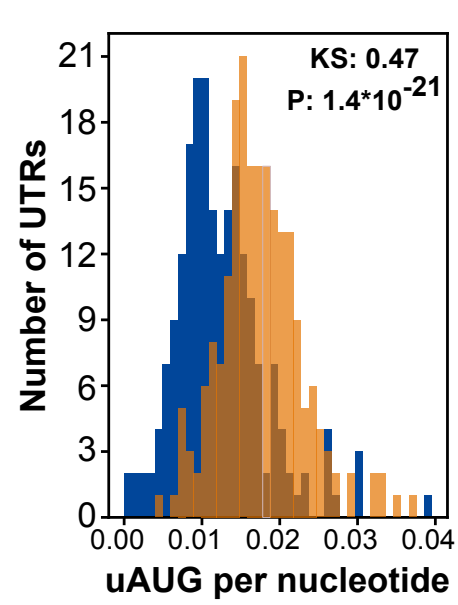

C

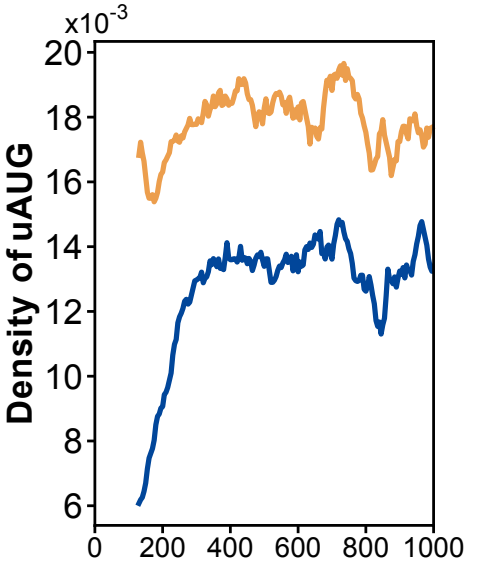

Distance from the start (nt)

D
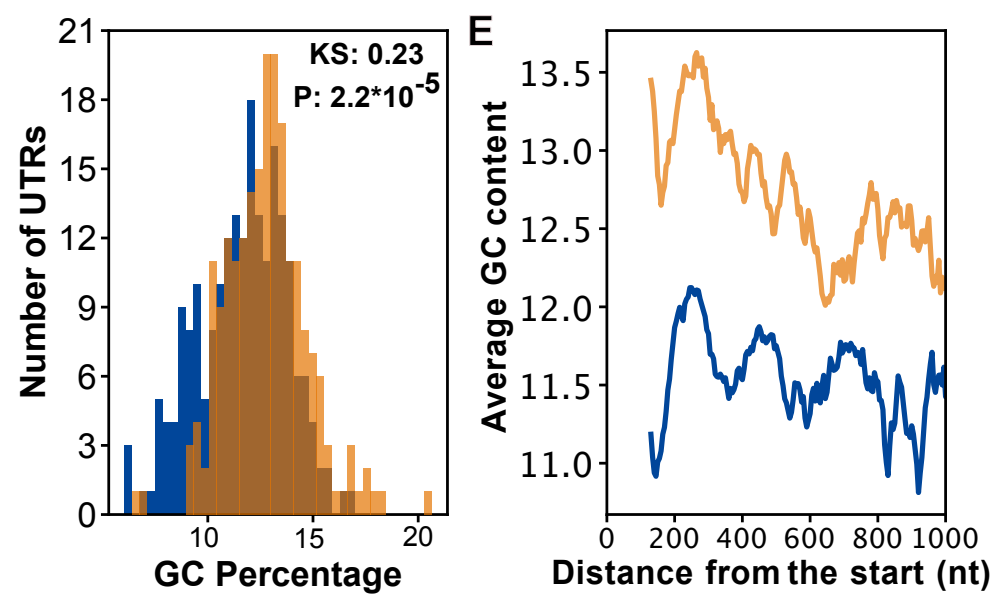
A

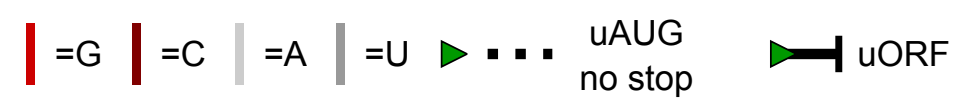

PF3D7_1428300: Active

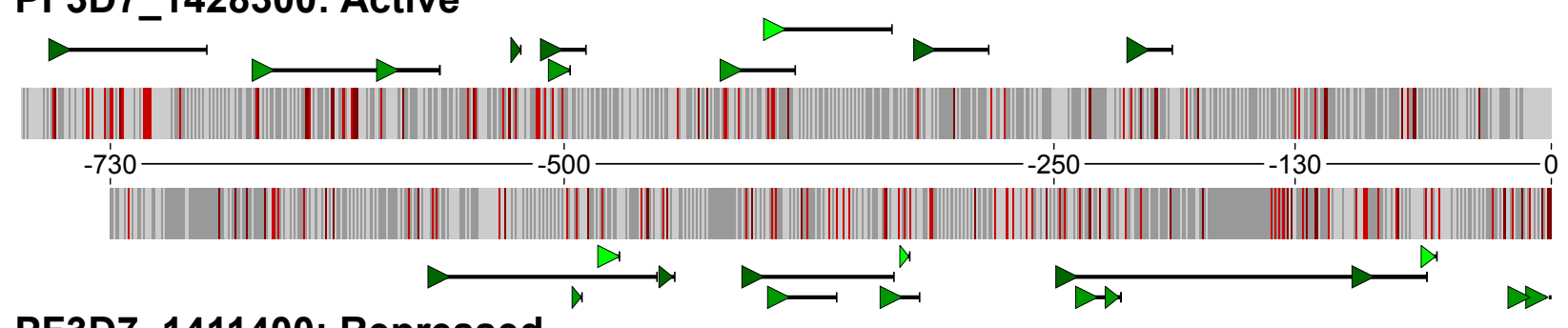

PF3D7_1411400: Repressed

B

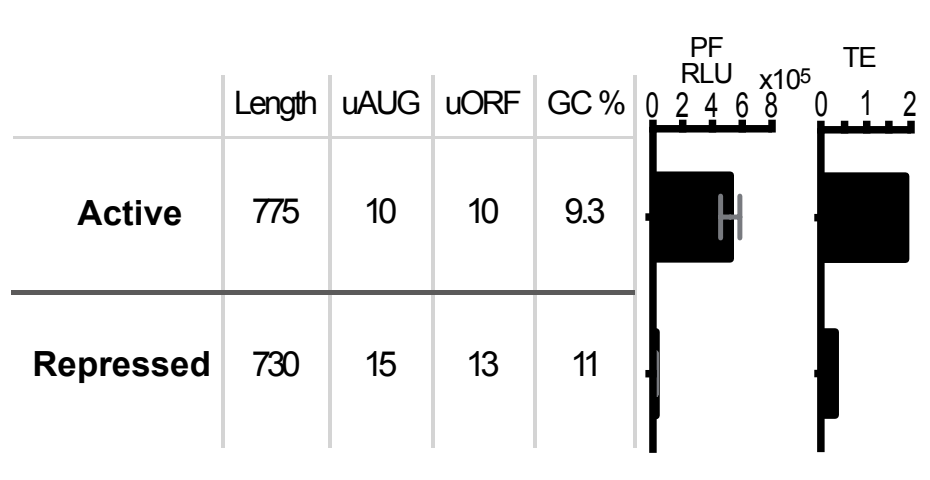

C

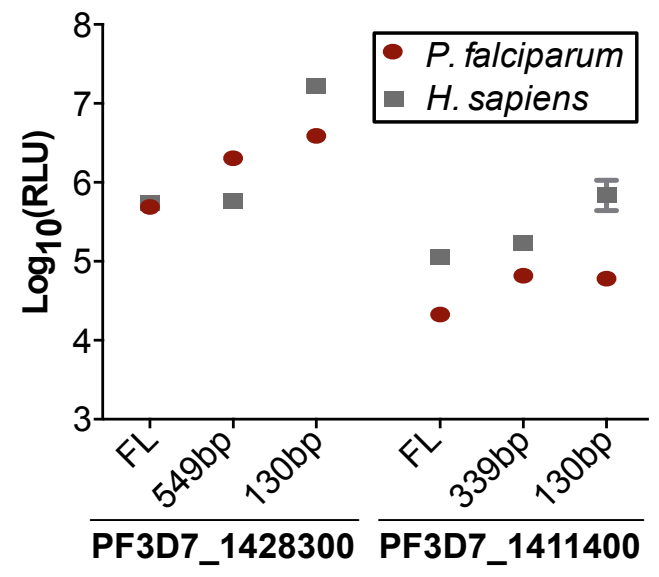

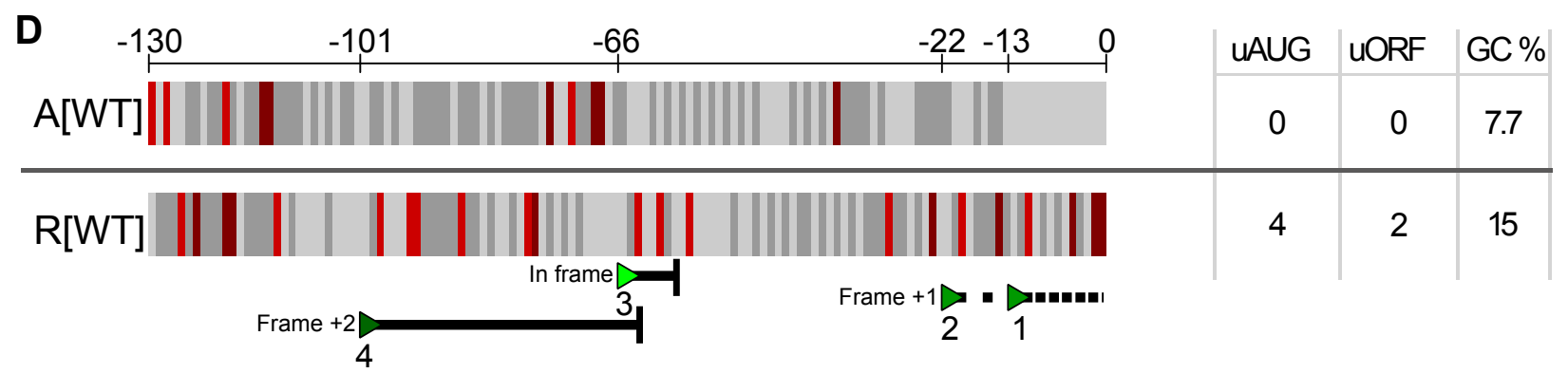


A

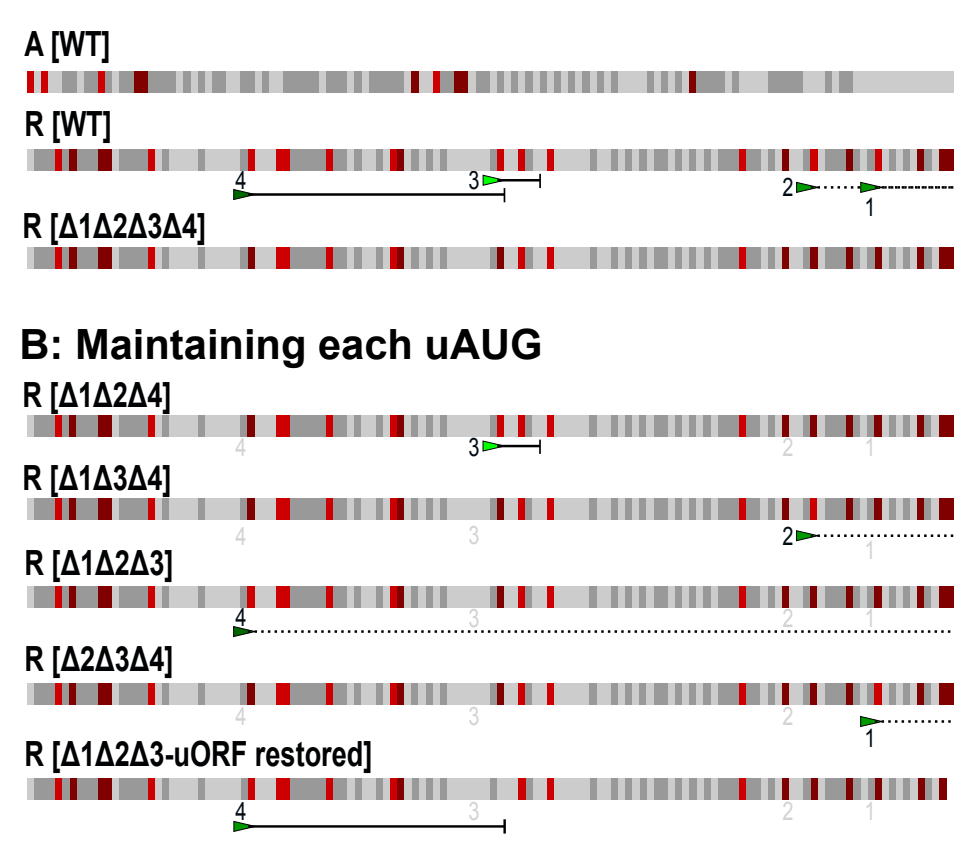

C: Removing pairs of uAUGs

$R[\Delta 1 \Delta 4]$

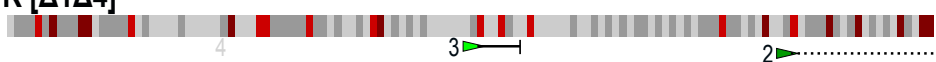

$\mathrm{R}[\Delta 2 \Delta 4]$

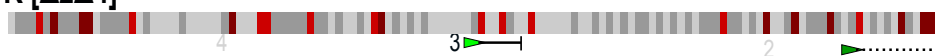

$R[\Delta 1 \Delta 3]$

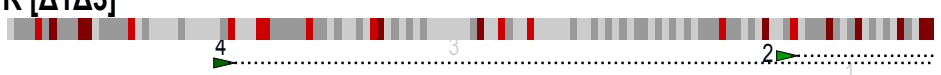

$\mathrm{R}[\Delta 3 \Delta 4]$

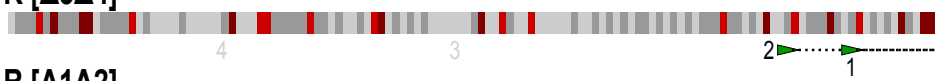

\section{$\mathrm{R}[\Delta 1 \Delta 2]$}

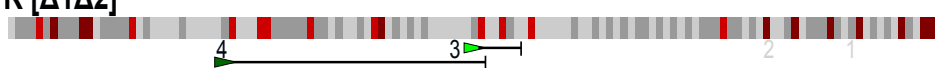

$\mathrm{R}[\Delta 2 \Delta 3]$

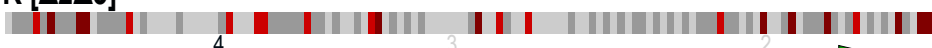

D: Removing individual UAUG

$\mathbf{R}[\Delta 1]$

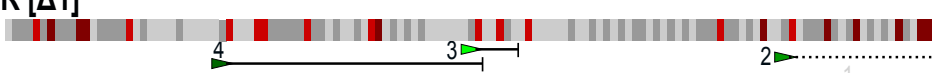

$\mathrm{R}[\Delta \mathbf{4}]$

R

\section{$R[\Delta 2]$}

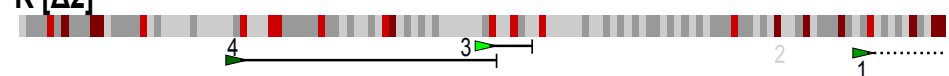

\section{$\mathrm{R}[\Delta 3]$}

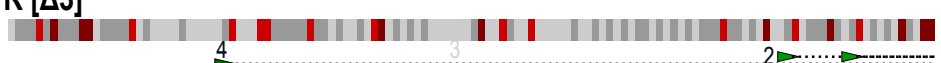

P. falciparum

$\log _{2}$ (value/R[WT])

$\begin{array}{lllllllll}-2 & -1 & 0 & 1 & 2 & 3 & 4 & 5 & 6\end{array}$
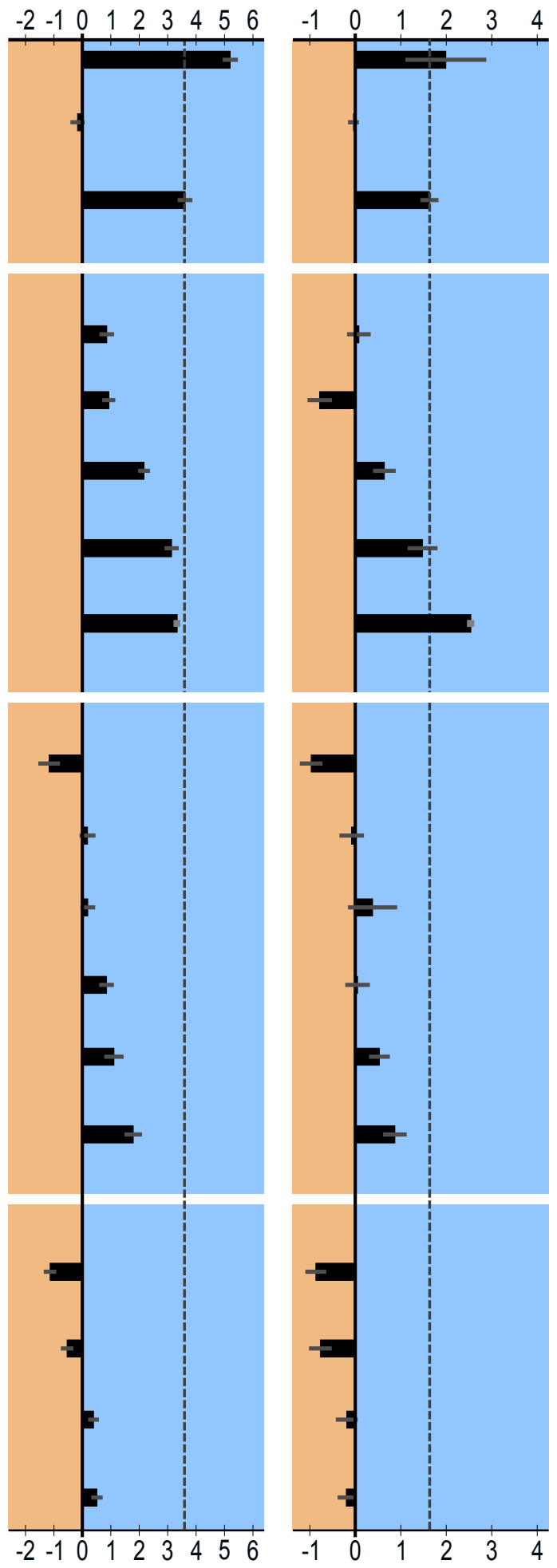
A

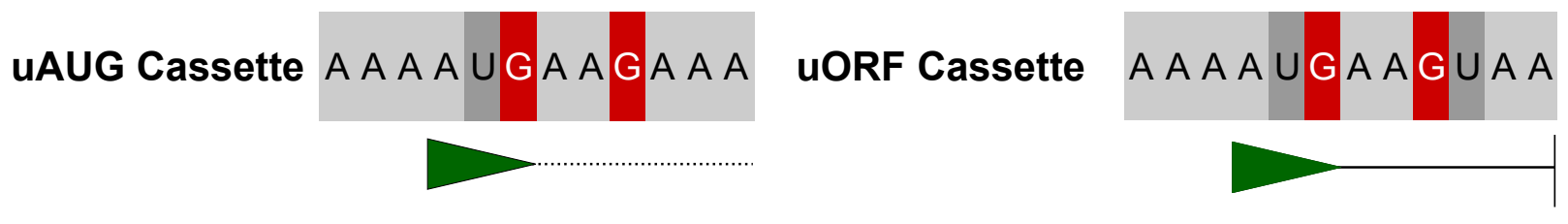

B

R[Cassette -14]

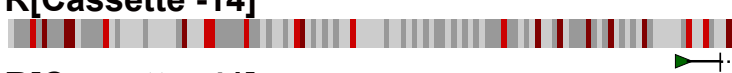

$R$ [Cassette -41]

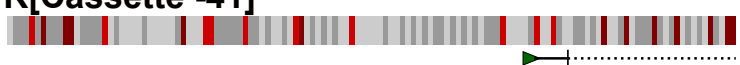

$R$ [Cassette -68]

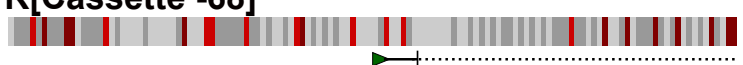

R[Cassette -95]

| II | || || | || | || |||||| | | ||||||||| | || | | | ||||||

$R$ [Cassette -122]

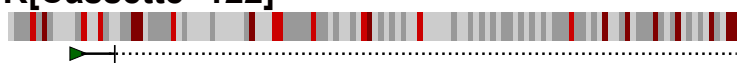

P. falciparum
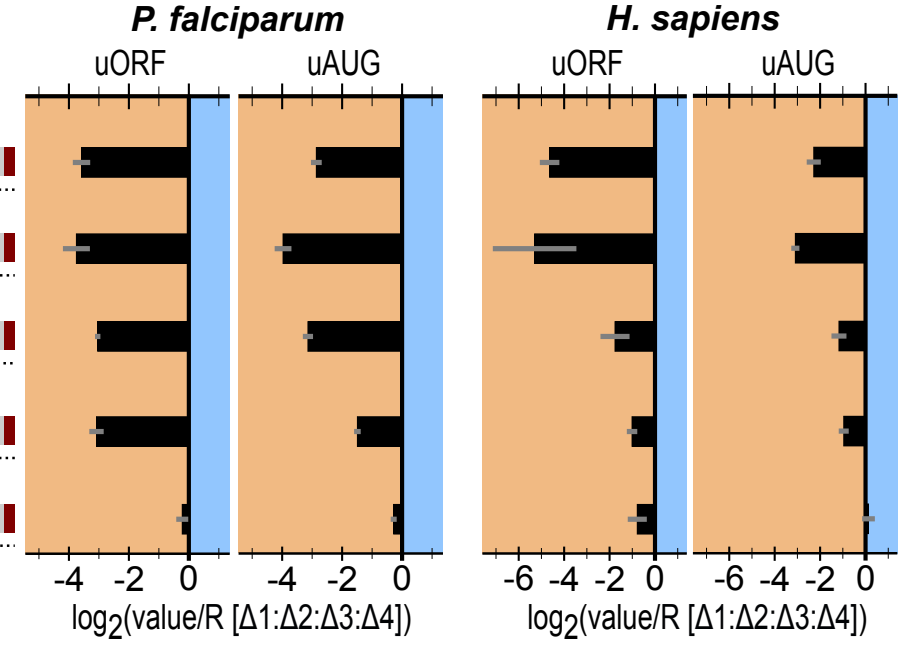
A A [WT] 1.7\% GC last $60 \mathrm{nt}$ II WI] $1.7 \%$ GC last 60 nt A [5\% last 60nt] A [10\% last 60nt] II A [20\% last $60 \mathrm{nt}]$ A [30\% last 60nt]

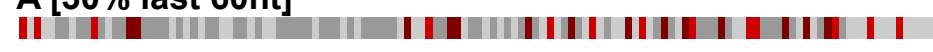

B

$R[\Delta 1: \Delta 2: \Delta 3: \Delta 4] 15 \%$ GC last $60 \mathrm{nt}$ $R[\Delta 1: \Delta 2: \Delta 3: \Delta 45 \%$ GC last $60 \mathrm{nt}]$ $R[\Delta 1: \Delta 2: \Delta 3: \Delta 40 \%$ GC last 60 nt $]$

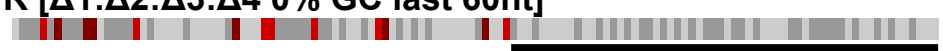
60 nucleotides
P. falciparum

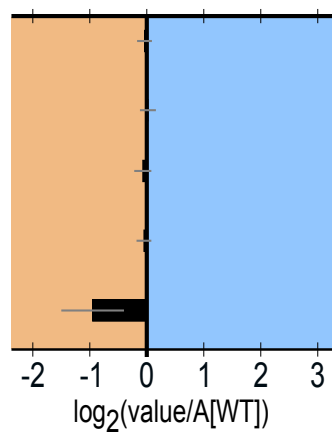

P. falciparum

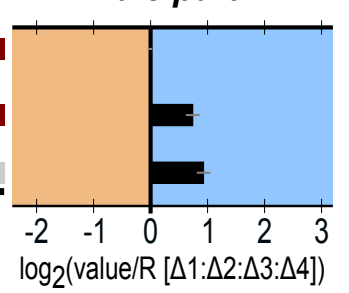

H. sapiens

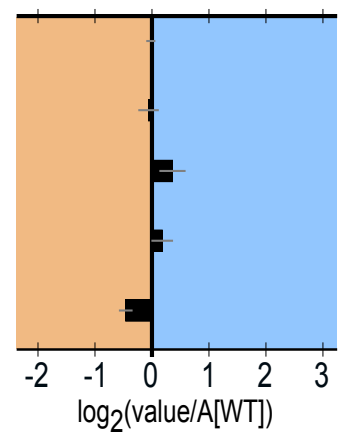

H. sapiens

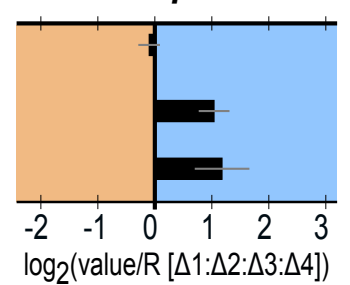


bioRxiv preprint doi: https://doi.org/10.1101/2021.09.07.459299; this version posted October 11, 2021. The copyright holder for this preprint (which was not certified by peer review) is the author/funder. All rights reserved. No reuse allowed without permission.

A) 3' end of R[WT] exchanged into A[WT]

A [WT]

R [WT]

A [20nt 3' of R]

IIIIII

A [40nt 3' of R]

A [60nt 3' of R]

II $[60$ it 3 of $R]$

B) 3' end of A[WT] exchanged into R[WT]

A [WT]

R1 [WT]

R [20nt 3' of A]

[II II A] IHIII

$R[60 n t$ 3' of $A]$

C) $5^{\prime}$ end of $R[\Delta 1: \Delta 2: \Delta 3: \Delta 4]$ exchanged into $A[W T]$

A [WT]

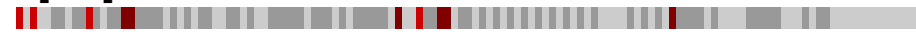
R [WT]

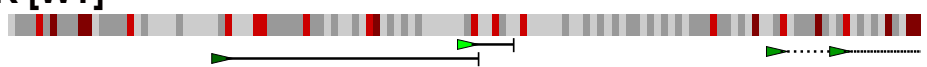

A [10nt 5' of R]

AII |

A [20nt 5' of R]

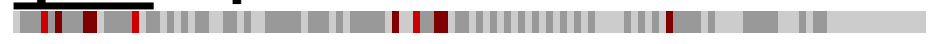

A [30nt 5' of R]

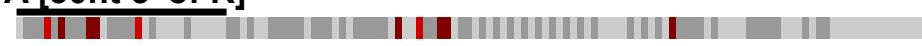

D) 5' end of A[WT] exchanged into $R[\Delta 1: \Delta 2: \Delta 3: \Delta 4]$

A [WT]

II |

$R[\Delta 1: \Delta 2: \Delta 3: \Delta 4]$

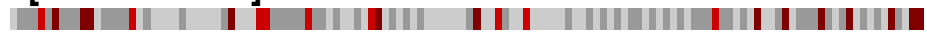

$R[\Delta 1: \Delta 2: \Delta 3: \Delta 410$ nt 5 ' of $A]$

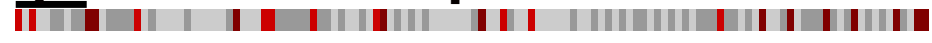

$R[\Delta 1: \Delta 2: \Delta 3: \Delta 420$ nt 5 ' of $A]$

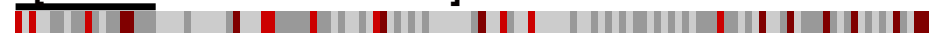

$R\left[\Delta 1: \Delta 2: \Delta 3: \Delta 430\right.$ nt $5^{\prime}$ of $\left.A\right]$

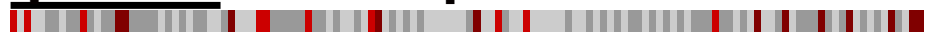
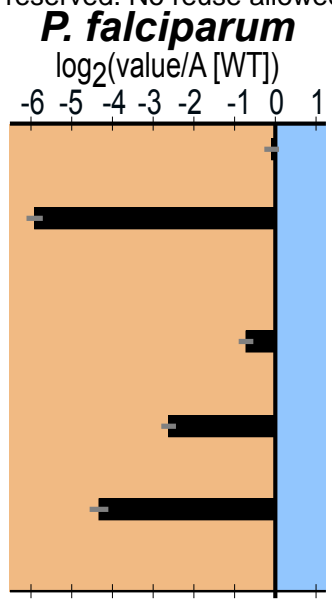

$\log _{2}($ value/R [WT])

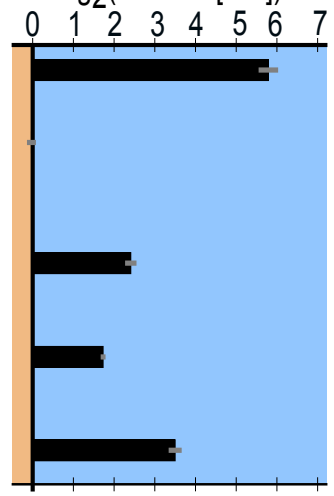

$\log _{2}$ (value/A [WT])

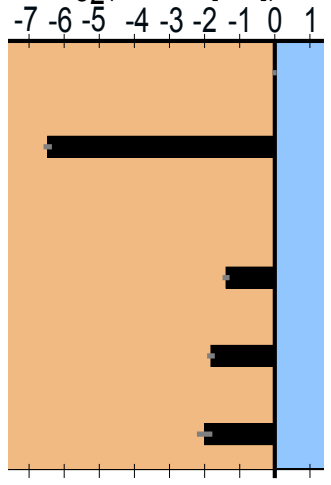

$\log _{2}$ (value/R [ $\left.\triangle 1: \Delta 2: \Delta 3: \Delta 4\right]$

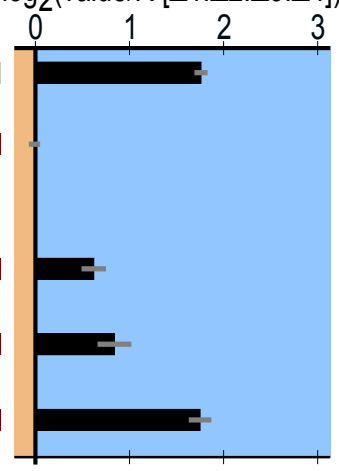

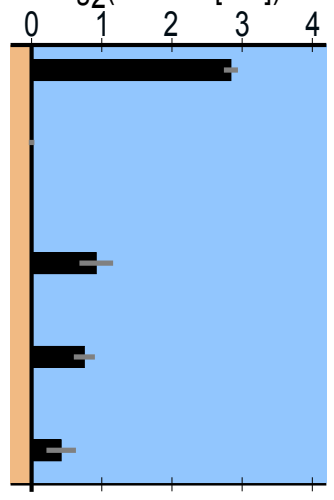

$\log _{2}$ (value/A [WT])

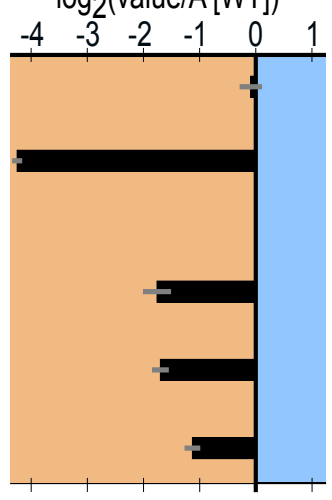

$\log _{2}$ (value/A [WT])

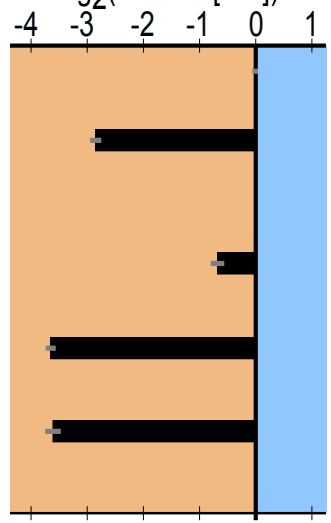

$\log _{2}($ value/R [WT])

$\log _{2}($ value/R $[\Delta 1: \Delta 2: \Delta 3: \Delta 4])$

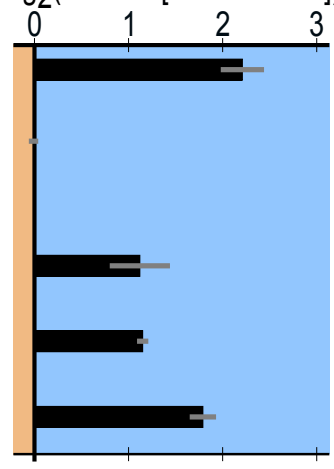


bioRxiv preprint doi: https://doi.org/10.1101/2021.09.07.459299; this version posted October 11, 2021. The copyright holder for this preprint (which was not certified by peer review) is the author/funder. All rights reserved. No reuse allowed without permission.

A

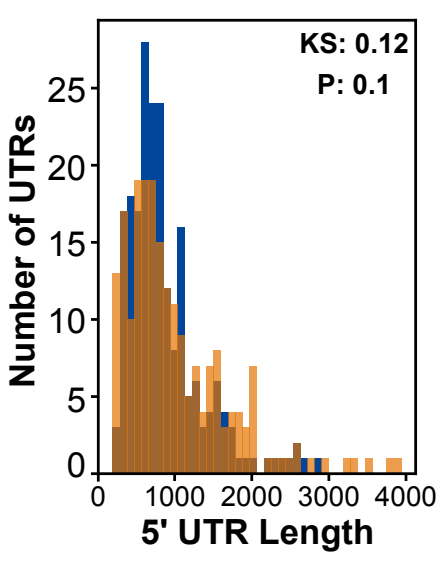

B

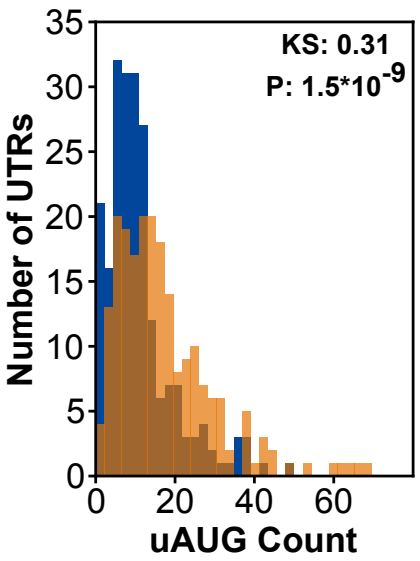




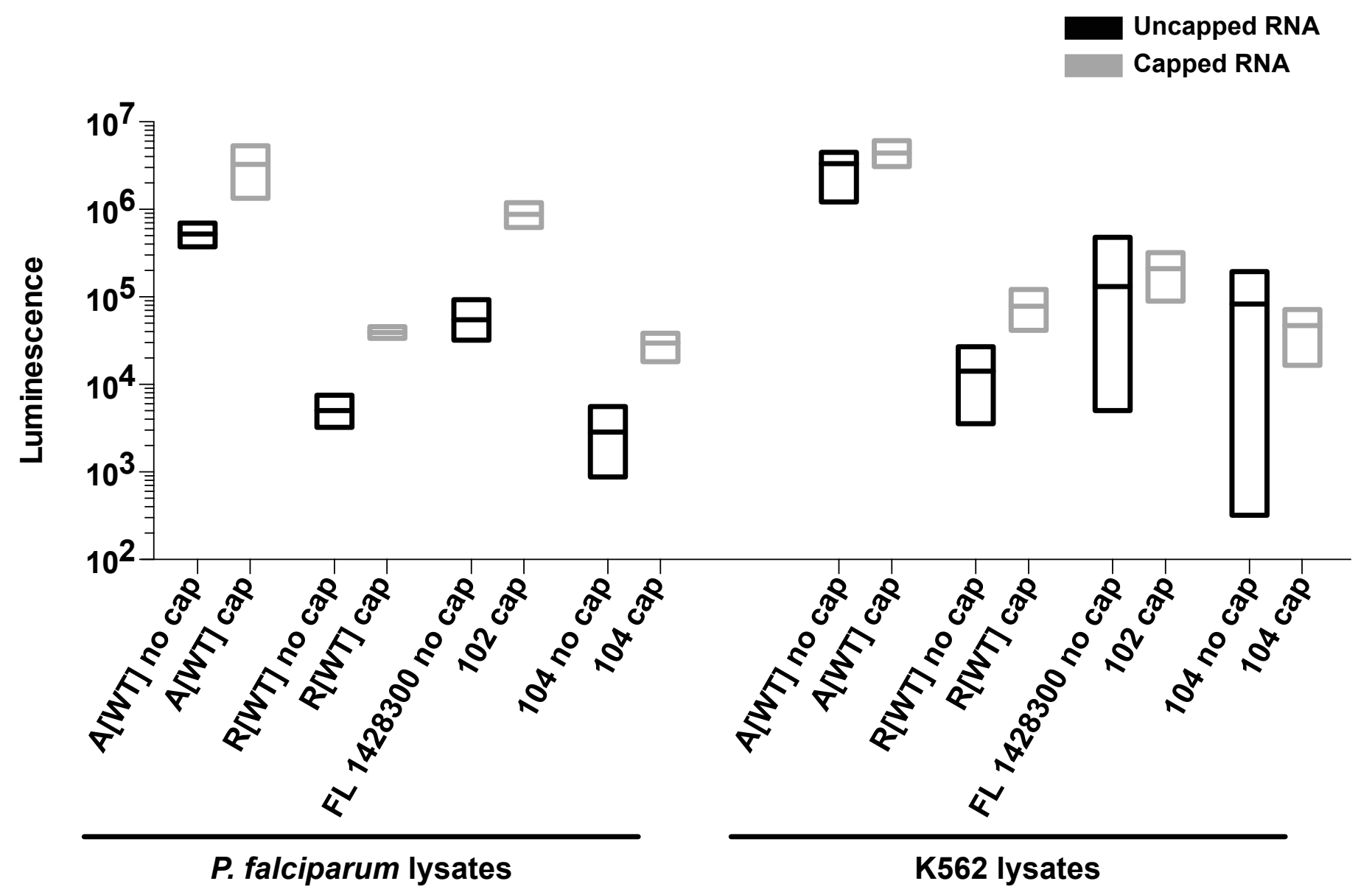




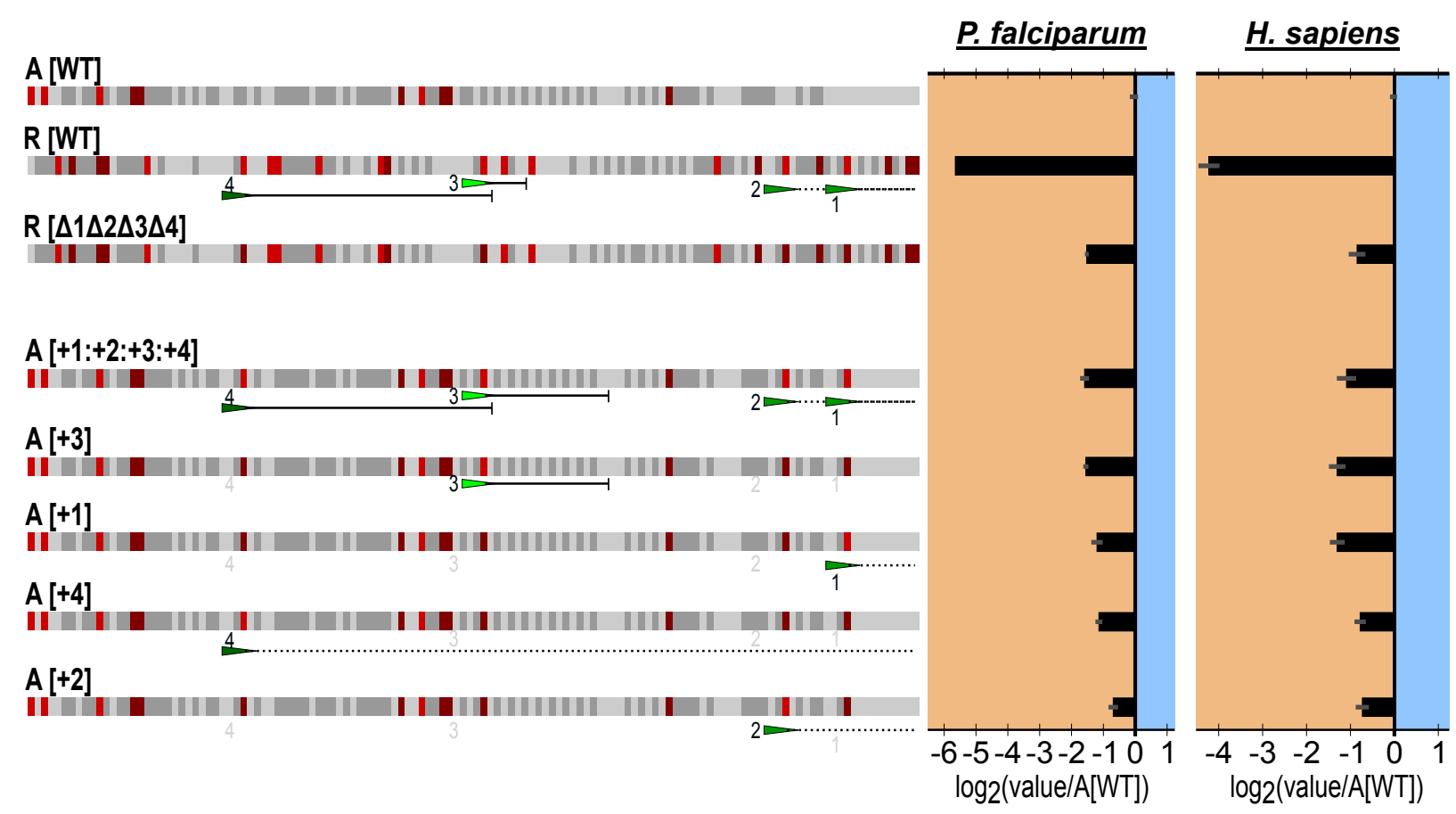


A

\section{$\%$ Maximum Signal}

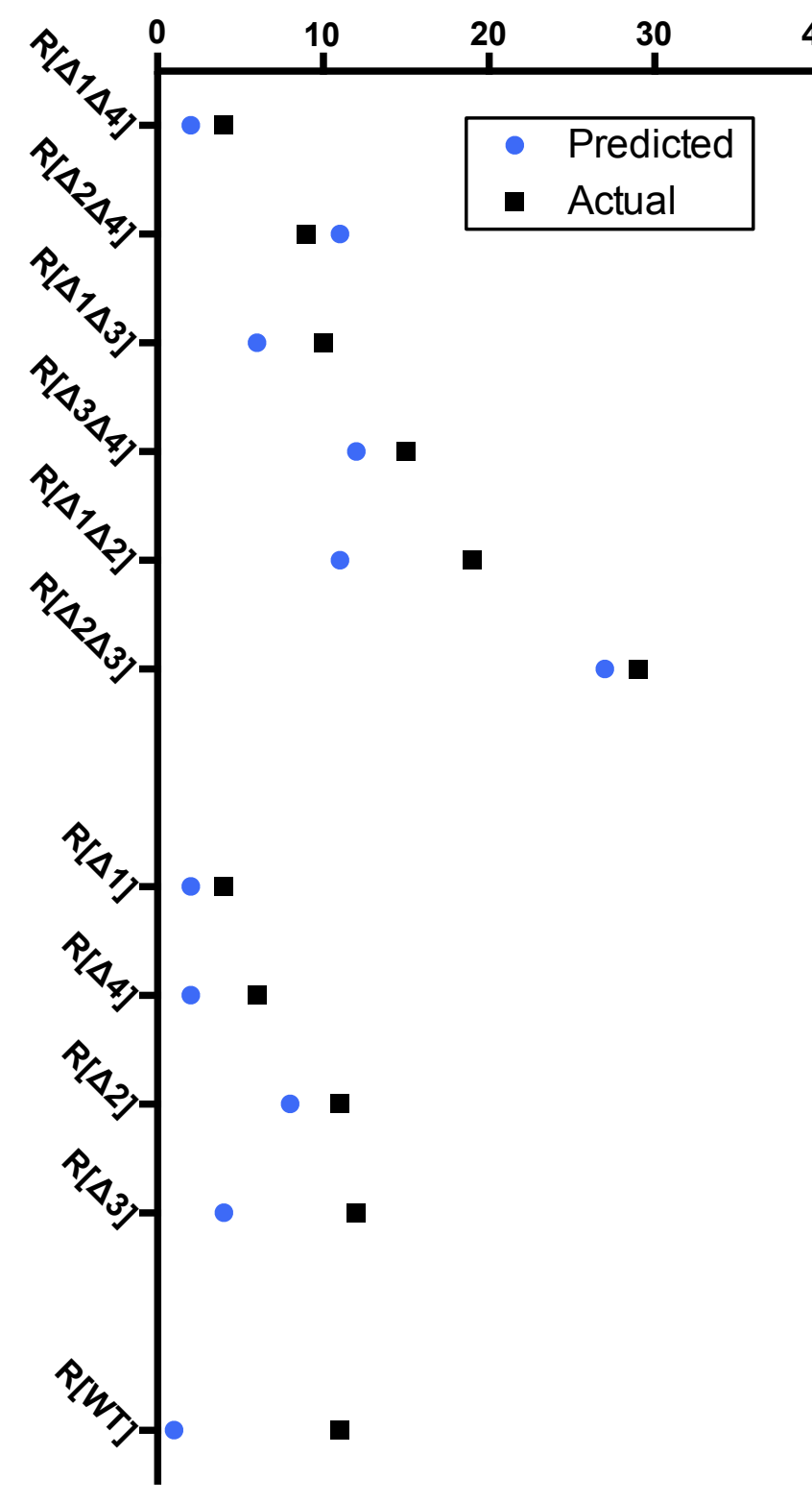

B $\%$ Maximum Signal

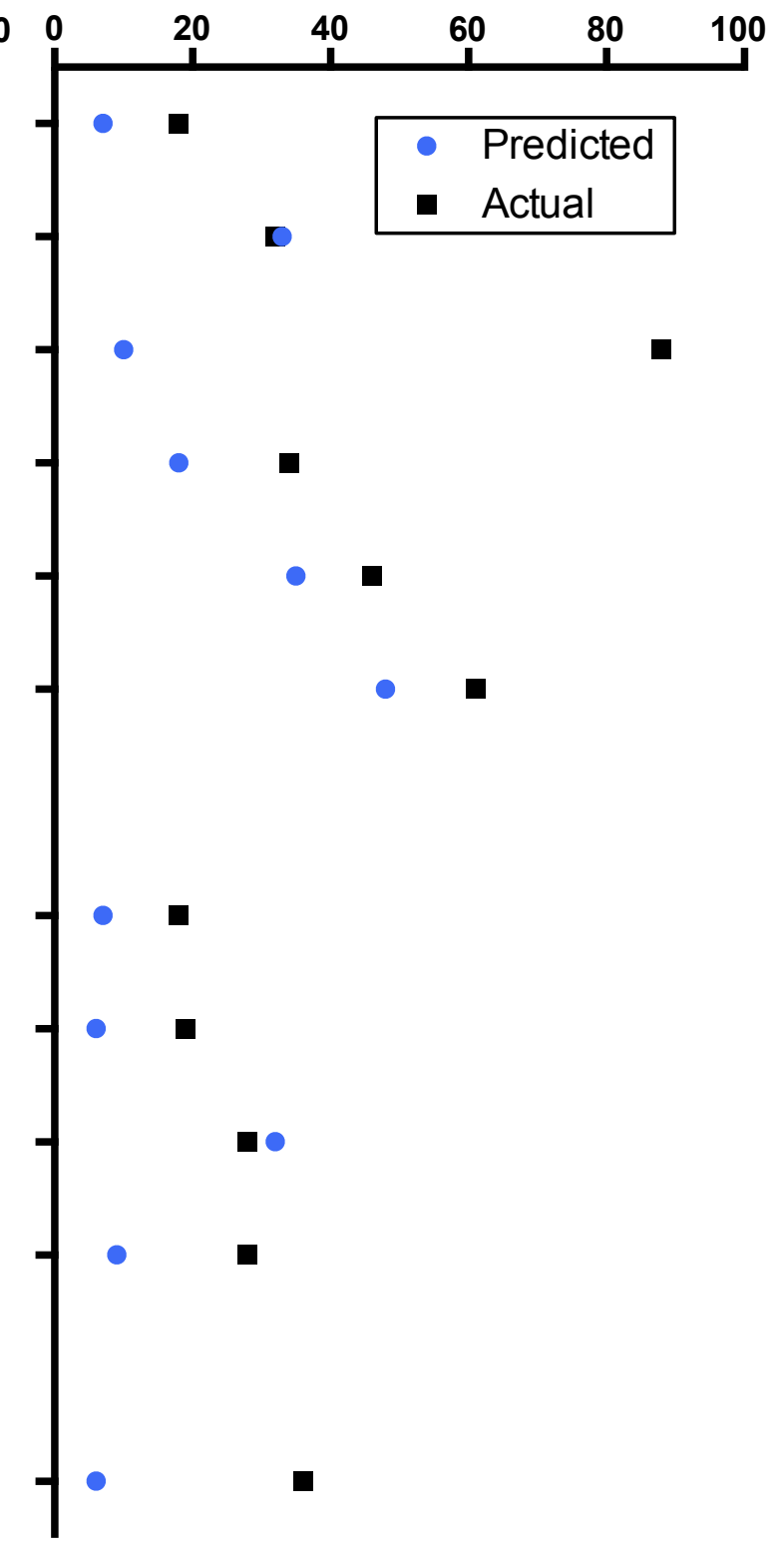

K562 


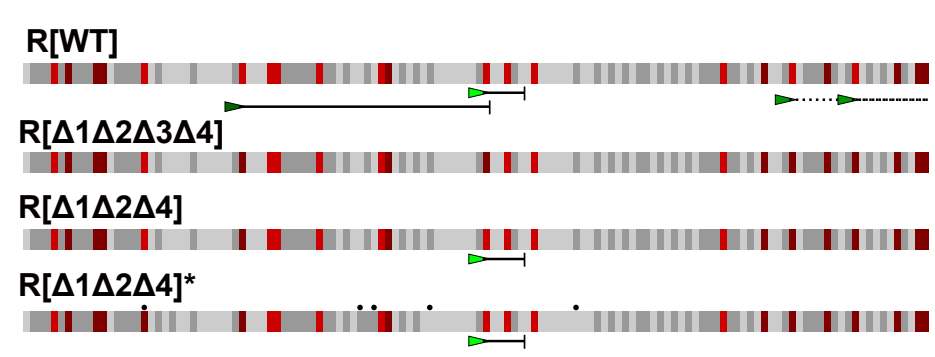

R[WT]

$R[\Delta 1 \Delta 2 \Delta 3 \Delta 4]$

$R[\Delta 1 \Delta 2 \Delta 4]$

$R[\Delta 1 \Delta 2 \Delta 4]^{*}$

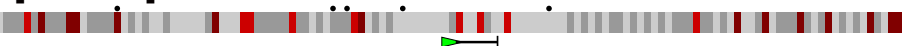

H. sapiens

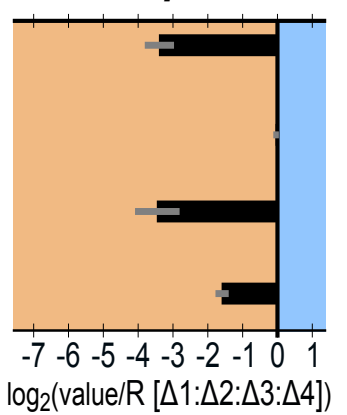



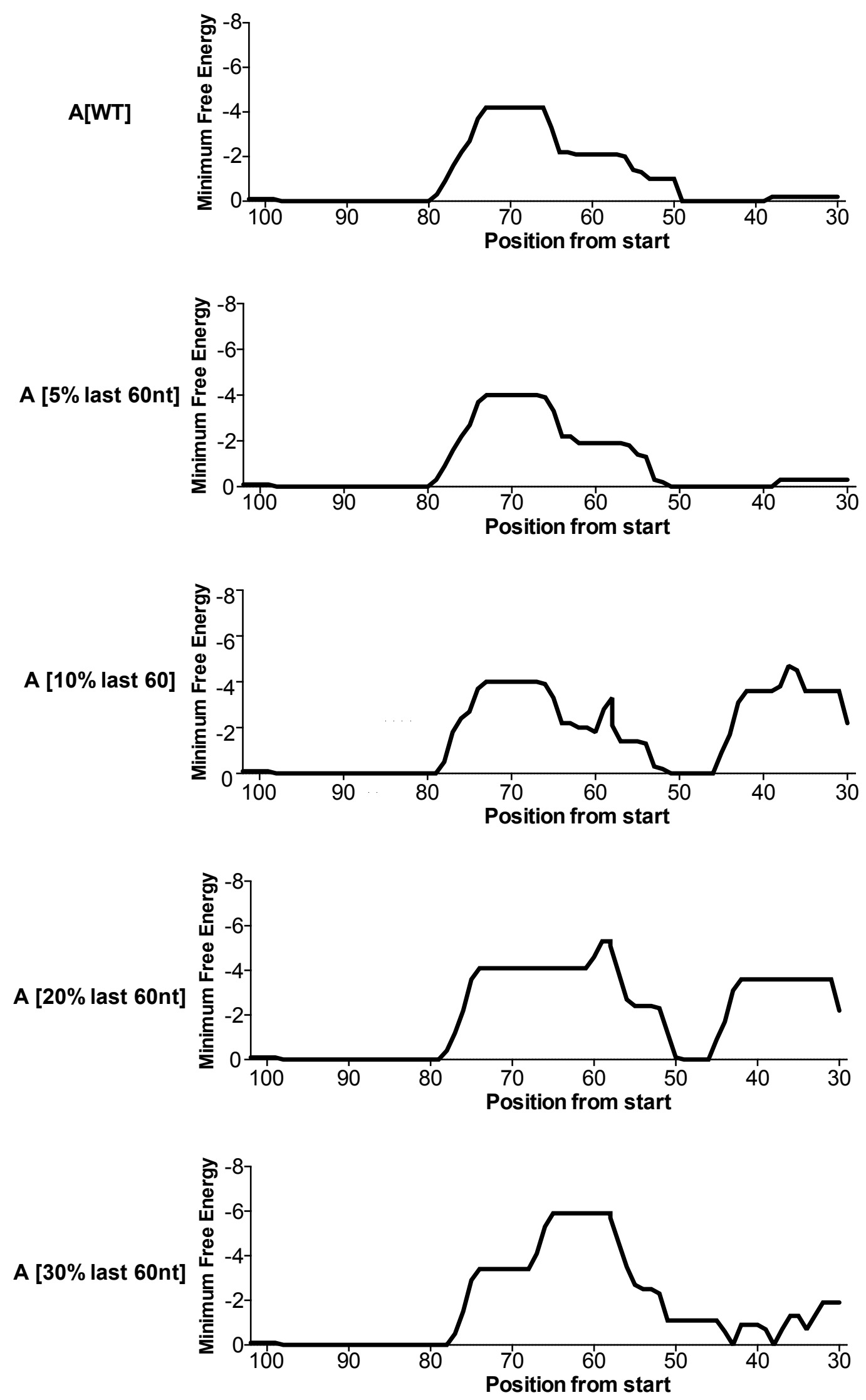